\title{
Coorbit Theory, Multi- $\alpha$-Modulation Frames, and the Concept of Joint Sparsity for Medical Multichannel Data Analysis
}

\author{
Stephan Dahlke, ${ }^{1}$ Gerd Teschke, ${ }^{2,3}$ and Krunoslav Stingl ${ }^{4}$ \\ ${ }^{1}$ FB 12 - Faculty of Mathematics and Computer Sciences, Philipps-University of Marburg, Hans-Meerwein-Street, \\ Lahnberge, 35032 Marburg, Germany \\ ${ }^{2}$ Institute for Computational Mathematics in Science and Technology, University of Applied Sciences Neubrandenburg, \\ Brodaer Street 2, 17033 Neubrandenburg, Germany \\ ${ }^{3}$ Zuse Institute Berlin, Takustrasse 7, 14195 Berlin-Dahlem, Germany \\ ${ }^{4}$ MEG-Center Tübingen, Otfried Müller Strasse 47, 72076 Tübingen, Germany
}

Correspondence should be addressed to Gerd Teschke, teschke@hs-nb.de

Received 30 November 2007; Revised 8 August 2008; Accepted 19 August 2008

Recommended by Qi Tian

This paper is concerned with the analysis and decomposition of medical multichannel data. We present a signal processing technique that reliably detects and separates signal components such as mMCG, fMCG, or MMG by involving the spatiotemporal morphology of the data provided by the multisensor geometry of the so-called multichannel superconducting quantum interference device (SQUID) system. The mathematical building blocks are coorbit theory, multi- $\alpha$-modulation frames, and the concept of joint sparsity measures. Combining the ingredients, we end up with an iterative procedure (with component-dependent projection operations) that delivers the individual signal components.

Copyright ( 2008 Stephan Dahlke et al. This is an open access article distributed under the Creative Commons Attribution License, which permits unrestricted use, distribution, and reproduction in any medium, provided the original work is properly cited.

\section{INTRODUCTION}

One focus in the field of prenatal diagnostics is the investigation of fetal developmental brain processes that are limited by the inaccessibility of the fetus. Currently, there exist two techniques for the study of fetal brain function in utero, namely, functional magnetic resonance imaging (fMRI) $[1,2]$ and fetal magnetoencephalography (fMEG) [3-6]. There are several advantages and disadvantages of both techniques. The fMEG, for instance, is a completely passive and noninvasive method with superior temporal resolution and is currently measured by a multichannel superconducting quantum interference device (SQUID) system, see Figure 1. However, the fMEG is measured in the presence of environmental noise and various near-field biological signals and other interference as, for example, maternal magnetocardiogram (mMCG), fetal magnetocardiogram (fMCG), uterine smooth muscle (magnetomyogram $=\mathrm{MMG})$, and motion artifacts $[7,8]$. After the removal of environmental noise [9], the emphasis is on the detection and separation of $\mathrm{mMCG}$, $\mathrm{MCG}$, and
MMG. Solving this detection problem seriously is the main prerequisite for observing and analyzing the fMEG. In the majority of reported work, the MCG was reduced by adaptive filtering and/or noise estimation techniques $[10,11]$. In [10], different algorithms for elimination of MCG from MEG recordings are considered, for example, direct subtraction (DS) of an MCG signal, adaptive interference cancellation (AIC), and orthogonal signal projection algorithms (OSPAs). All these approaches and their slightly modified versions are used for fMEG detection. In this paper, we present a different data processing technique that reliably detects both the mMCG + fMCG and MMG + "motion artifacts" by involving the spatiotemporal morphology of the data given by the multisensor geometry information. Mathematically, the main ingredients of our procedure are the so-called multi- $\alpha$-modulation frames (for which the construction relies on the theory of coorbit spaces) for an optimal/sparse signal expansion and the concept of joint sparsity measures.

A sparse representation of an element in a Hilbert or Banach space is a series expansion with respect to an 


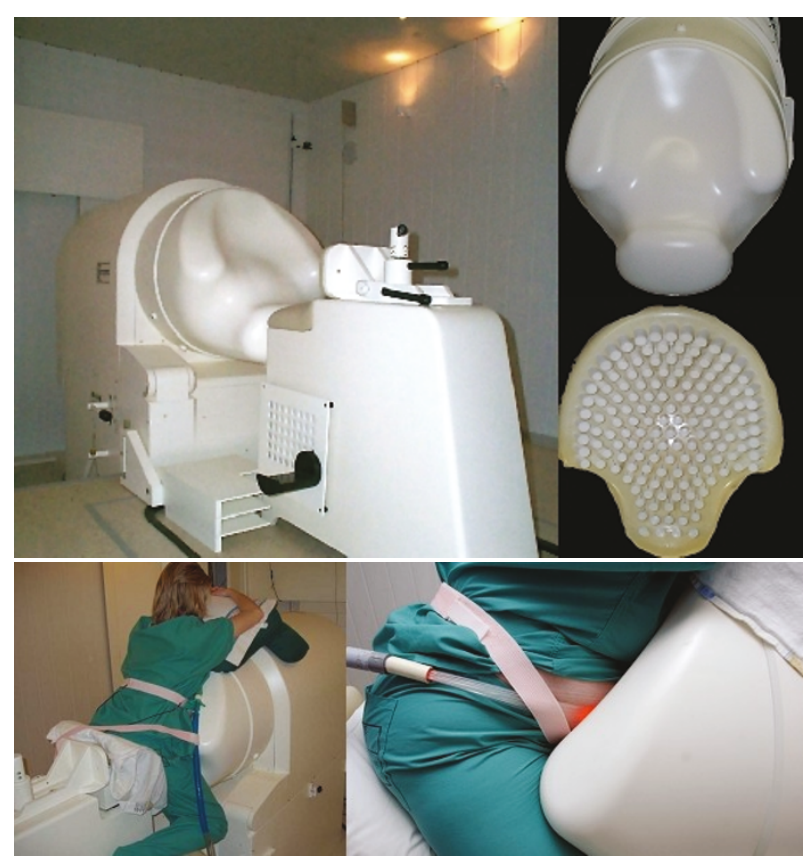

FIGURE 1: Multichannel superconducting quantum interference device (SQUID) system.

orthonormal basis or a frame that has only a small number of large/nonzero coefficients. Several types of signals appearing in nature admit sparse frame expansions, and thus sparsity is a realistic assumption for a very large class of problems. Recent developments have shown the practical impact of sparse signal reconstruction (even the possibility to reconstruct sparse signals from incomplete information [12-14]). This is in particular the case for the medical multichannel data under consideration that usually consist of pattern representing specific biomedical information (mMCG and fMCG). But multichannel signals (i.e., vector valued functions) may not only possess sparse frame expansions for each channel individually, but additionally (and this is the novelty) the different channels can also exhibit common sparsity patterns. The mMCG and AMCG exhibit a very rich morphology that appears in all the channels at the same temporal locations. This will be reflected, for example, in sparse wavelet/Gabor expansions $[15,16]$ with relevant coefficients appearing at the same labels, or in turn in sparse gradients with supports at the same locations. Hence, an adequate sparsity constraint is the so-called common or joint sparsity measure that promotes patterns of multichannel data that do not belong only to one individual channel but to all of them simultaneously.

In order to sparsely represent the MCG data, we propose the usage of multi- $\alpha$-modulation frames. These frames have only been recently developed as a mixture of Gabor and wavelet frames. Wavelet frames are optimal for piecewise smooth signals with isolated singularities, whereas Gabor frames have been very successfully applied to the analysis of periodic structures. Therefore, the $\alpha$-modulation frames have the potential to detect both features at the same time, so they seem to be extremely well suited for the problems studied in this paper. Indeed, the numerical experiments presented here definitely confirm this conjecture.

This paper is organized as follows. In Section 2, we briefly recall the setting of $\alpha$-modulation frames as far as this is needed for our purposes. Then, in Section 3, we explain how these frames can be used in multichannel data processing involving joint sparsity constraints. Finally, in Section 4, we present the numerical experiments.

\section{COORBIT THEORY AND $\alpha$-MODULATION FRAMES}

In this section, we review the basic that provides the socalled $\alpha$-modulation frames. We propose to treat the medical data analysis problem with this specific kind of frame expansions since varying the parameter $\alpha$ allows to switch between completely different frame expansions highlighting different features of the signal to be analyzed while having to manage only one frame construction principle. The focus is not yet on multichannel data approximation but rather on the basic methodologies that apply for single-channel signals but can simply be extended to multichannel data (in Section 3).

In general, the motivation (and central issue in applied analysis) is the problem of analyzing and approximating a given signal. The first step is always to decompose the signal with respect to a suitable set of building blocks. These building blocks may, for example, consist of the elements of a basis, a frame, or even of the elements of huge dictionaries. Classical examples with many important practical applications are wavelet bases/frames and Gabor frames, respectively. The wavelet transform is very useful to analyze piecewise smooth signals with isolated singularities, whereas the Gabor transform is well-suited for the analysis of periodic structures such as textures. Quite surprisingly, there is a common thread behind both transforms, and that is a group theory. In general, a unitary representation $U$ of a locally compact group $G$ in a Hilbert space $\mathscr{H}$ is called square integrable if there exists a function $\psi \in \mathscr{H}$ such that

$$
\int_{G}\left|\langle\psi, U(g) \psi\rangle_{\mathscr{H}}\right|^{2} d \mu(g)<\infty
$$

where $d \mu$ denotes the (left) Haar measure on $G$. In this case, the voice transform

$$
V_{\psi} f(g):=\langle f, U(g) \psi\rangle_{\mathscr{H}}
$$

is well defined and invertible on its range by its adjoint. It turns out that the Gabor transform can be interpreted as the voice transform associated with a representation of the WeylHeisenberg group in $L_{2}$, whereas the wavelet transform is related with a square-integrable representation of the affine group in $L_{2}$.

Since both transforms have their specific advantages, it is quite natural to try to combine them in a joint transform. 


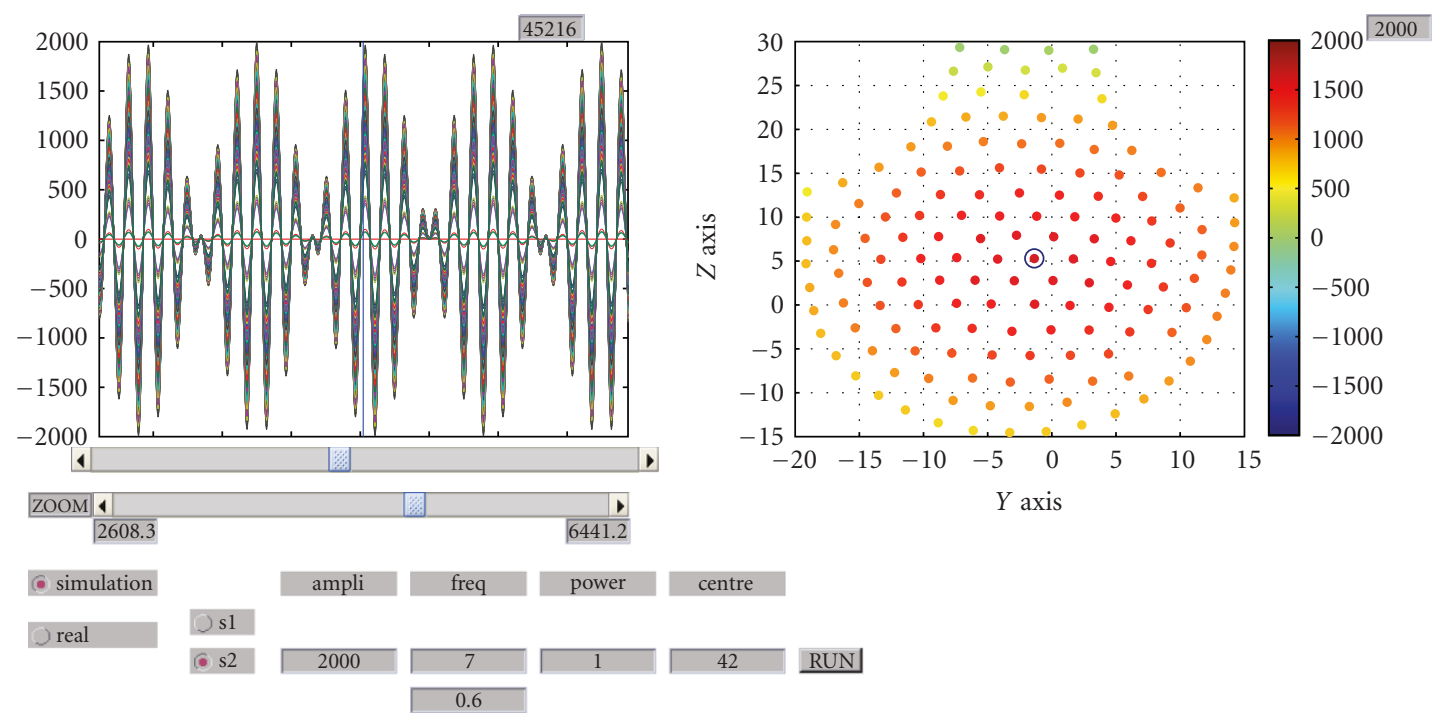

FIGURE 2: Left: second component, generated by combination of two sinusoidal functions $(7 \mathrm{~Hz}$ and $0.6 \mathrm{~Hz})$. The different amplitudes correspond to signals of the different channels. Right: geometric visualization of the SQUID device with 151 sensors (coils). The color encodes the Gaussian weighting, that is, the influence of the synthetic background signal. The center of appearance of the synthetic signal is marked by a circle.

One way to achieve this would be to use the affine WeylHeisenberg group $G_{\mathrm{aWH}}$ which is the set $\mathbb{R}^{2+1} \times \mathbb{R}_{+}$equipped with group law

$$
\begin{aligned}
& (q, p, a, \varphi) \circ\left(q^{\prime}, p^{\prime}, a^{\prime}, \varphi^{\prime}\right) \\
& \quad=\left(q+a q^{\prime}, p+a^{-1} p^{\prime}, a a^{\prime}, \varphi+\varphi^{\prime}+p a q^{\prime}\right) .
\end{aligned}
$$

This group has the Stone-Von-Neumann representation on $L_{2}(\mathbb{R})$ as follows:

$$
\begin{aligned}
U(q, p, a, \varphi) f(x) & =a^{-1 / 2} e^{2 \pi i(p(x-q)+\varphi)} f\left(\frac{x-q}{a}\right) \\
& =e^{2 \pi i \varphi} T_{x} M_{\omega} D_{a} f(t),
\end{aligned}
$$

where

$$
\begin{gathered}
M_{\omega} f(t)=e^{2 \pi i \omega t} f(t), \quad T_{x} f(t)=f(t-x), \\
D_{a} f(t)=|a|^{-1 / 2} f\left(\frac{t}{a}\right),
\end{gathered}
$$

which obviously contains all three basic operations, that is, dilations, modulations, and translations. Unfortunately, $U$ is not square integrable. One way to overcome this problem is to work with representations modulo quotients. In general, given a locally compact group $G$ with closed subgroup $H$, we consider the quotient group $X=G / H$ and fix a section $\sigma: X \rightarrow G$. Then, we define the generalized voice transform

$$
V_{\psi} f(x):=\langle f, U(\sigma(x)) \psi\rangle_{\mathcal{H}} .
$$

In the case of the affine Weyl-Heisenberg group, it has been shown in [17] that by using the specific group $H:=$ $\left\{(0,0, a, \varphi) \in G_{\mathrm{aWH}}\right\}$ and the specific section $\sigma(x, \omega)=$ $(x, \omega, \beta(x, \omega), 0), \beta(x, \omega)=(1+|\omega|)^{-\alpha}, \alpha \in[0,1)$, the associated voice transform (6) is indeed well defined and invertible on its range. Hence, it gives rise to a mixed form of the wavelet and the Gabor transform, and it also provides some kind of homotopy between both cases. Indeed, for $\alpha=0$, we are in the classical Gabor setting, whereas the case $\alpha=1$ is very close to the wavelet setting (see, e.g., [17] for details).

Once a square-integrable representation modulo quotient is established, there is also a natural way to define associated smoothness spaces, the so-called coorbit spaces, by collecting all functions for which the voice transform has a certain decay, see [18-20]. More precisely, given some positive measurable weight function $v$ on $X$ and $1 \leq p \leq \infty$, let

$$
L_{p, v}(X):=\left\{f \text { measurable }: f v \in L_{p}(X)\right\}
$$

Then, for suitable $\psi$, we define the spaces

$$
\begin{aligned}
\mathscr{H}_{p, v} & :=\left\{f: V_{\psi}\left(A_{\sigma}^{-1} f\right) \in L_{p, v}\right\}, \\
A_{\sigma} f & :=\int_{X}\langle f, U(\sigma(x)) \psi\rangle_{\mathscr{H}} U(\sigma(x)) \psi d \mu,
\end{aligned}
$$

where $d \mu$ denotes a quasi-invariant measure on $X$. In the classical cases, that is, for the affine group and the WeylHeisenberg group, one obtains the Besov spaces and the modulation spaces, respectively. In the setting of the affine Weyl-Heisenberg group and the specific case $v_{s}(\omega)=(1+$ $|\omega|)^{s}$, the following theorem has been shown in [17]. 

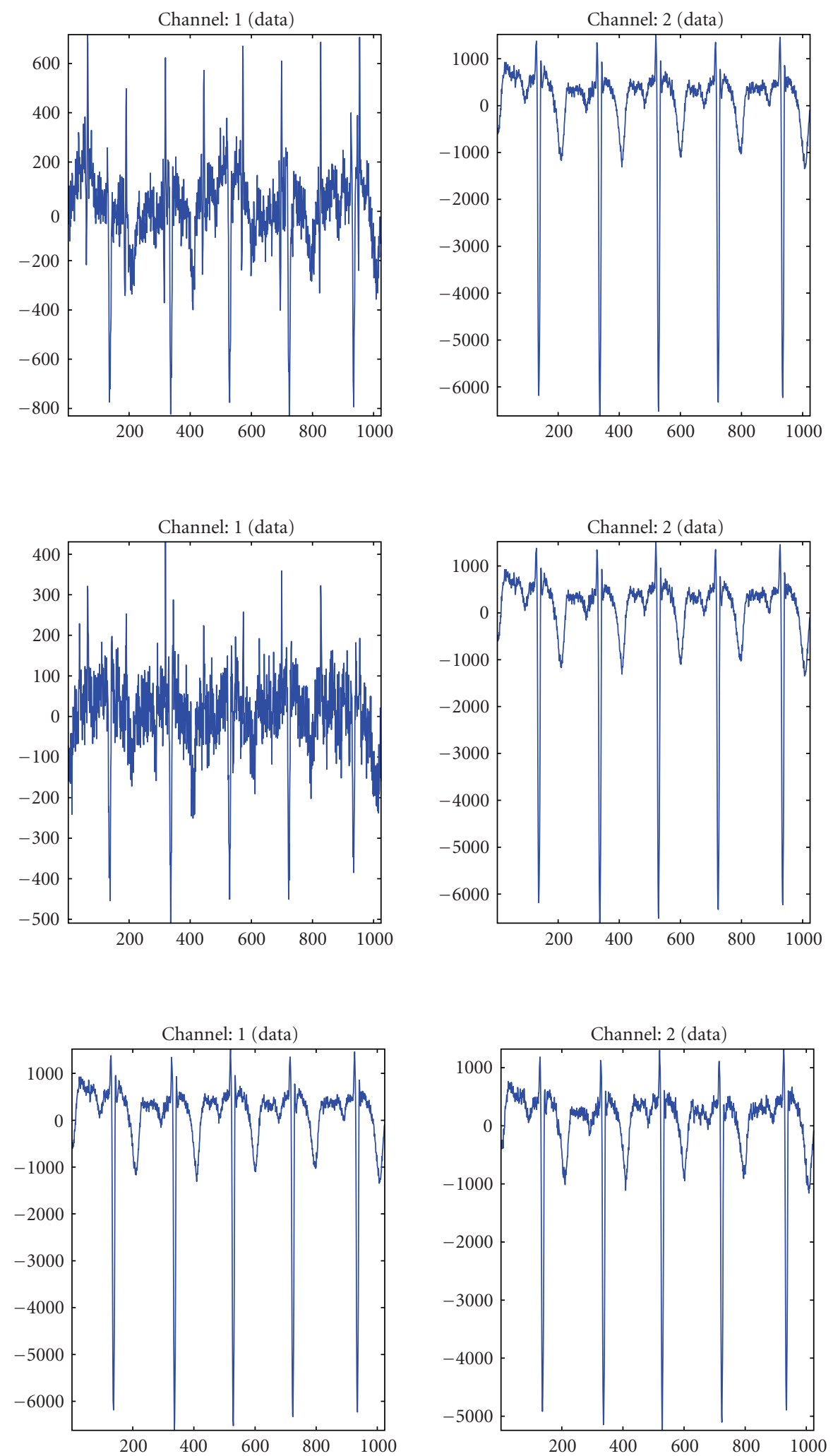

FIgURe 3: Measured spontaneous activity of selected individual channels. Top row: channel 1 corresponds to coil number 20, and channel 2 corresponds to coil number 40. Middle row: channel 1 corresponds to coil number 80, and channel 2 corresponds to coil number 40 . Bottom row: channel 1 corresponds to coil number 40 , and channel 2 corresponds to coil number 41 . It can be clearly observed that the neighboring channels have similar structures, whereas channels with large geometric distance have completely different structures. 

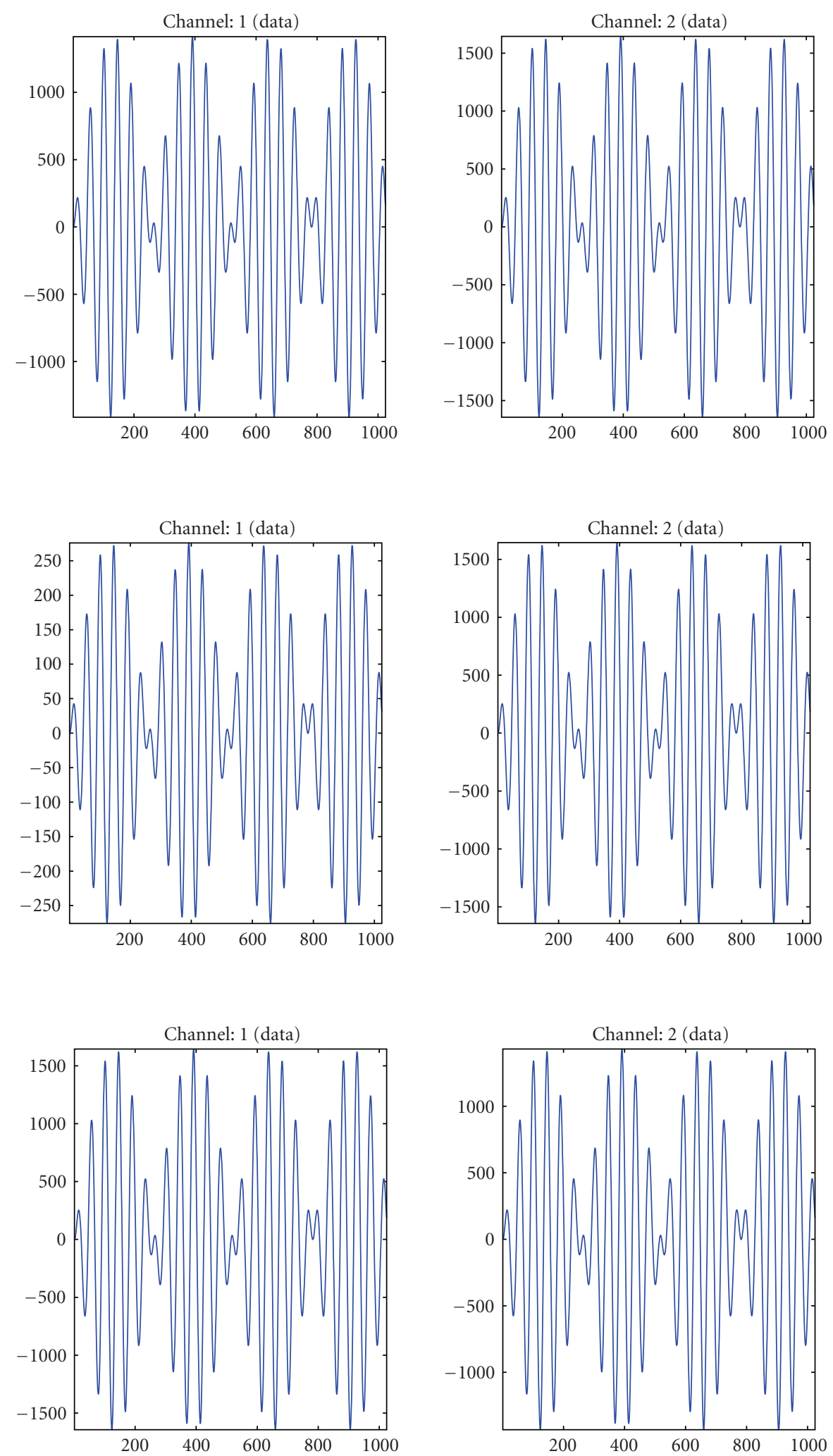

FIgURE 4: Synthetic sinusoidal signals of selected individual channels. Top row: channel 1 corresponds to coil number 20, and channel 2 corresponds to coil number 40. Middle row: channel 1 corresponds to coil number 80, and channel 2 corresponds to coil number 40. Bottom row: channel 1 corresponds to coil number 40, and channel 2 corresponds to coil number 41 . Due to the Gaussian weighting, the neighboring channels have similar amplitudes, whereas channels with large geometric distance have significantly different amplitudes (attenuation). 

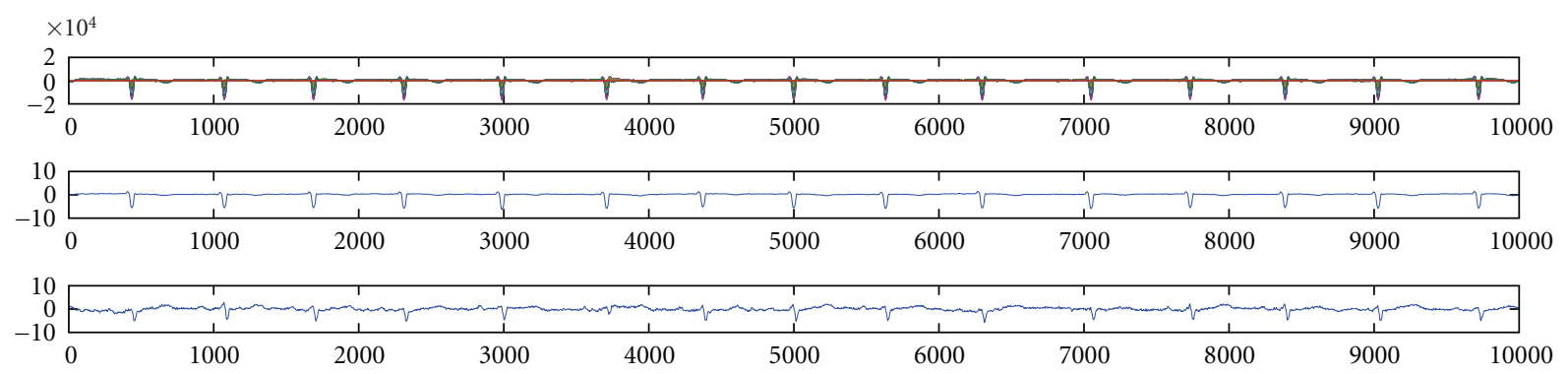

-5
0
0
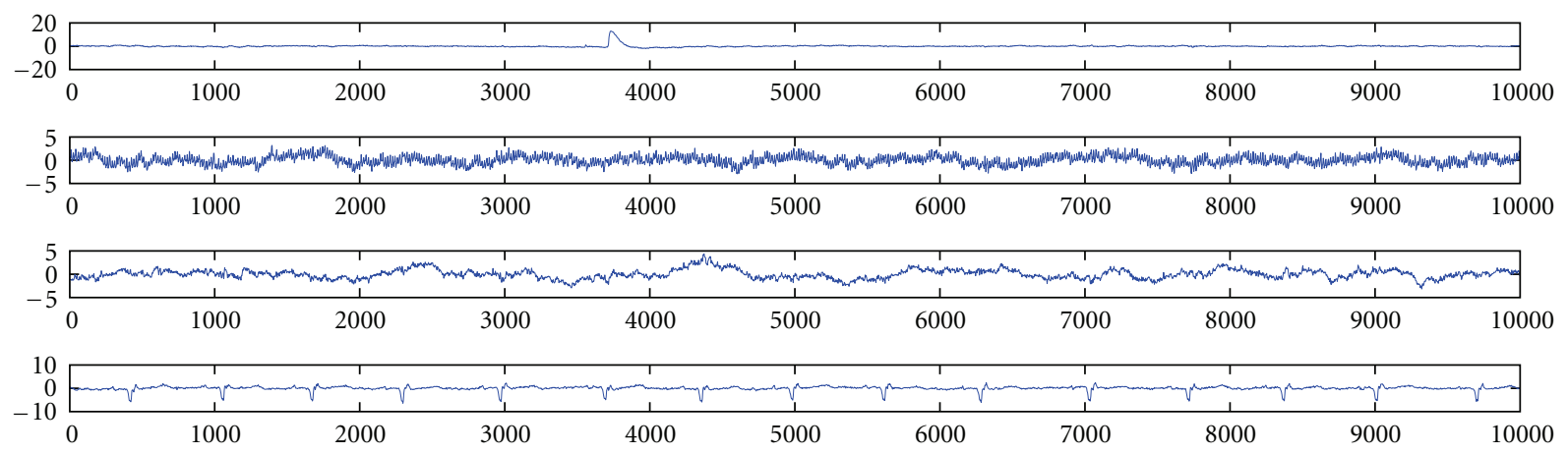

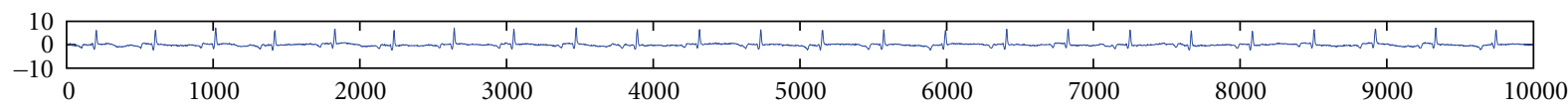
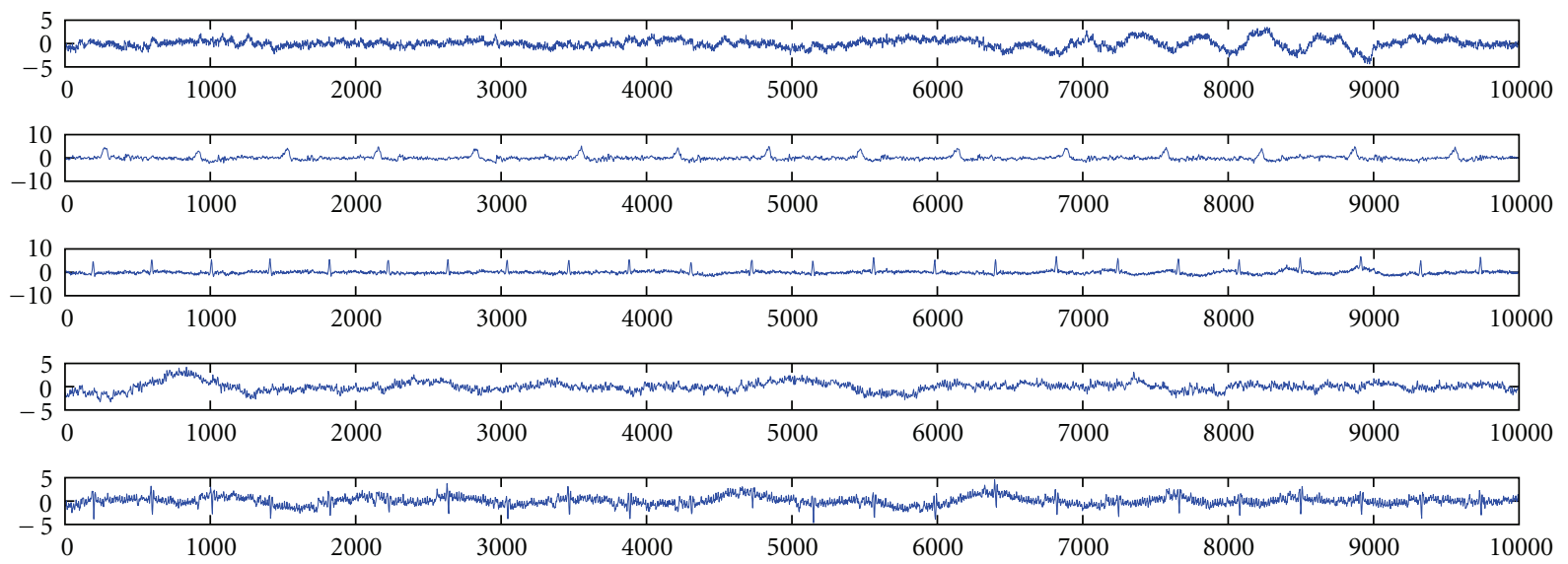

FIGURE 5: Top row: signal to be analyzed. Second to bottom row: ICA decomposition of generated signal "spontaneous activity + sinusoidal signal," where the maximum amplitude of the synthetic signal component is $125 \mathrm{fT}$.

Theorem 1. Let $1 \leq p \leq \infty, 0 \leq \alpha<1$, and $s \in \mathbb{R}$. Let $\psi \in L_{2}$ with supp $\hat{\psi}$ compact and $\hat{\psi} \in C^{2}$. Then the coorbit

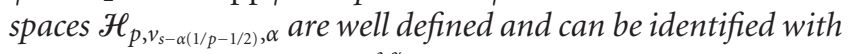
the $\alpha$-modulation spaces $M_{p, p}^{s, \alpha}$, which are defined by

$M_{p, p}^{s+\alpha(1 / q-1 / 2), \alpha}(\mathbb{R})=\left\{f \in \mathcal{S}^{\prime}(\mathbb{R}):\langle f, U(\sigma(x, \omega)) \psi\rangle \in L_{p \cdot v_{s}}\left(\mathbb{R}^{2}\right)\right\}$.

Consequently, the $\alpha$-modulation spaces are the natural smoothness spaces associated with representations modulo quotients of the affine Weyl-Heisenberg group.
When it comes to practical applications, then one can only work with discrete data, and therefore it is necessary to discretize the underlying representation in a suitable way. Indeed, in a series of papers [18-20], Feichtinger and Gröchenig have shown that a judicious discretization gives rise to frame decompositions. The general setting can be described as follows. Given a Hilbert space $\mathcal{H}$, a countable set $\left\{f_{n}: n \in \mathbb{N}\right\}$ is called a frame for $\mathscr{H}$ if

$$
\|f\|_{\mathscr{H}}^{2} \sim \sum_{n \in \mathbb{N}}\left|\left\langle f, f_{n}\right\rangle_{\mathscr{H}}\right|^{2} \quad \forall f \in \mathscr{H} .
$$



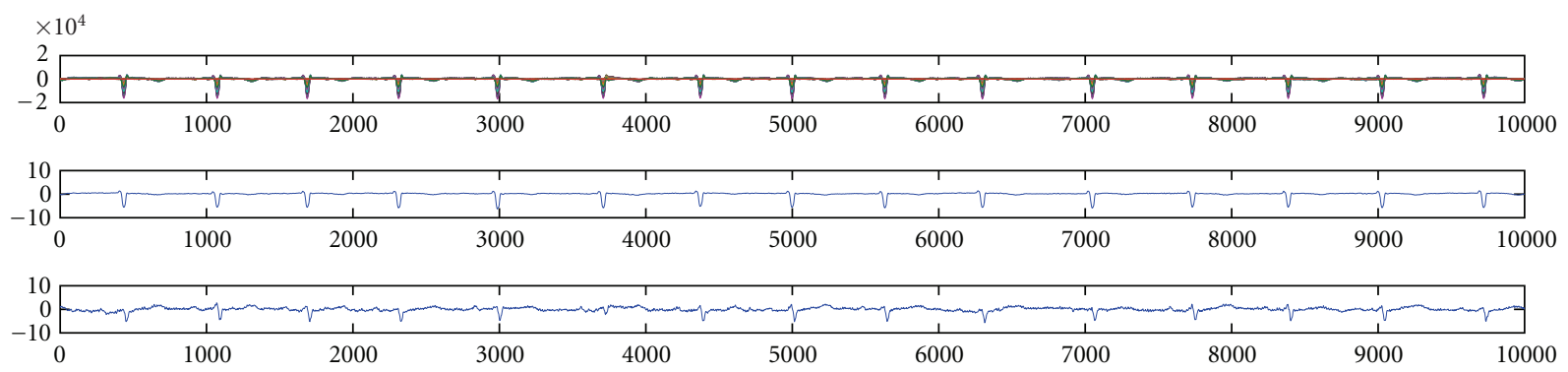

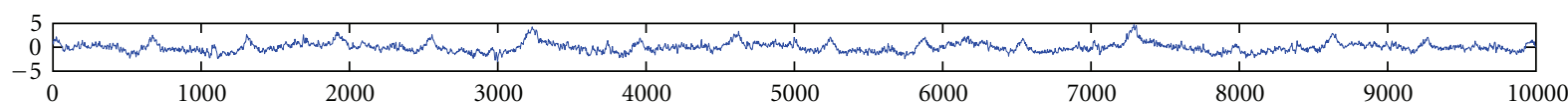
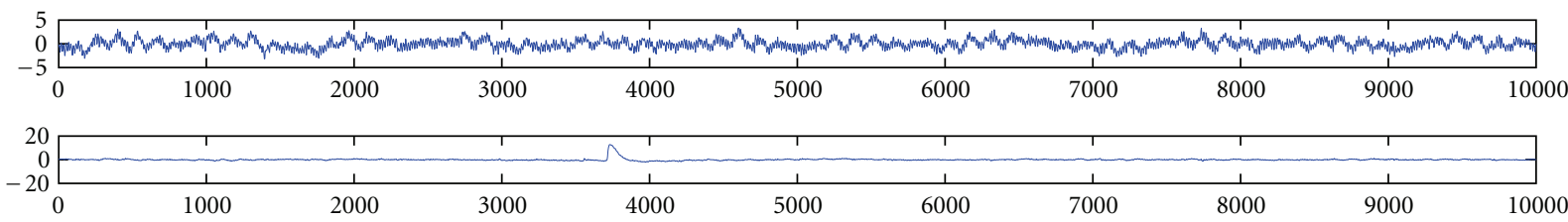

$=10$ (10
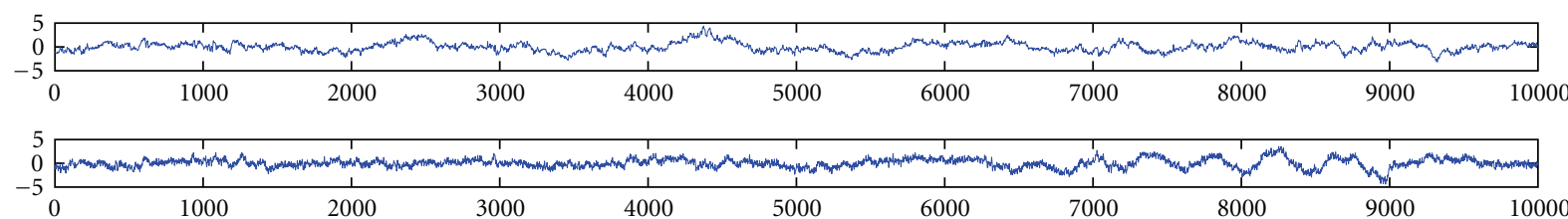

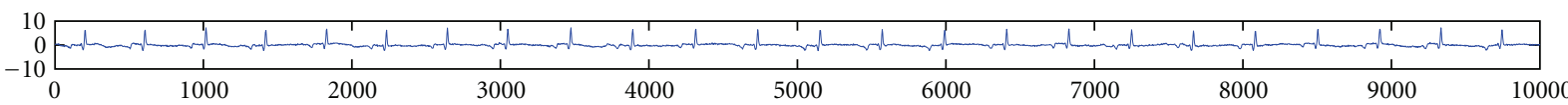

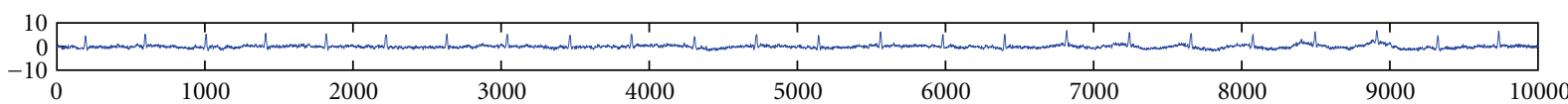

$\begin{array}{lllllllll}10 & 0 \\ 0 & 1000 & 2000 & 3000 & 4000 & 5000 & 6000 & 7000 & 8000\end{array}$
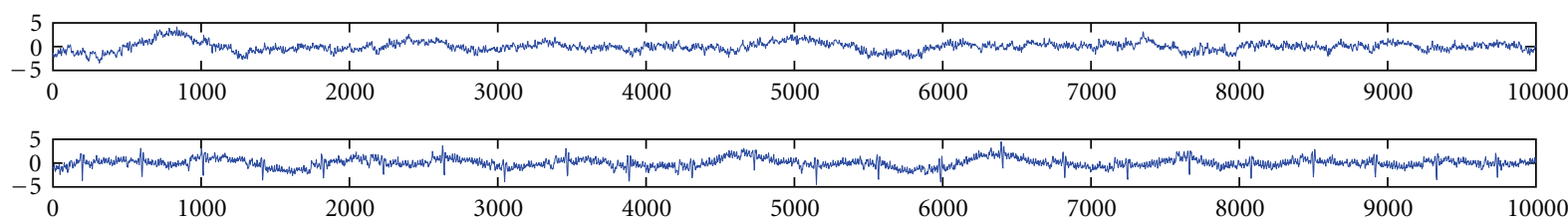

FIgURE 6: Top row: signal to be analyzed. Second to bottom row: ICA decomposition of generated signal "spontaneous activity + sinusoidal signal," where the maximum amplitude of the synthetic signal component is $250 \mathrm{fT}$.

As a consequence of (10), the corresponding operators of analysis and synthesis given by

$$
\begin{gathered}
F: \mathcal{H} \longrightarrow \ell_{2}(\mathbb{N}), \quad f \longmapsto\left(\left\langle f, f_{n}\right\rangle_{\mathscr{H}}\right)_{n \in \mathbb{N}}, \\
F^{*}: \ell_{2} \longrightarrow \mathcal{H}, \quad \mathbf{c} \longmapsto \sum_{n \in \mathbb{N}} c_{n} f_{n}
\end{gathered}
$$

are bounded. The composition $S:=F^{*} F$ is boundedly invertible and gives rise to the following decomposition and reconstruction formulas:

$$
\begin{aligned}
f & =S S^{-1} f=\sum_{n \in \mathbb{N}}\left\langle f, S^{-1} f_{n}\right\rangle_{\mathscr{H}} f_{n} \\
& =S^{-1} S f=\sum_{n \in \mathbb{N}}\left\langle f, f_{n}\right\rangle_{\mathscr{H}} S^{-1} f_{n} .
\end{aligned}
$$

The Feichtinger-Gröchenig theory gives rise to frame decompositions of this type, not only for the underlying representation space $\mathcal{H}$ but also for the associated coorbit spaces. Indeed, it is possible to decompose any element in the 

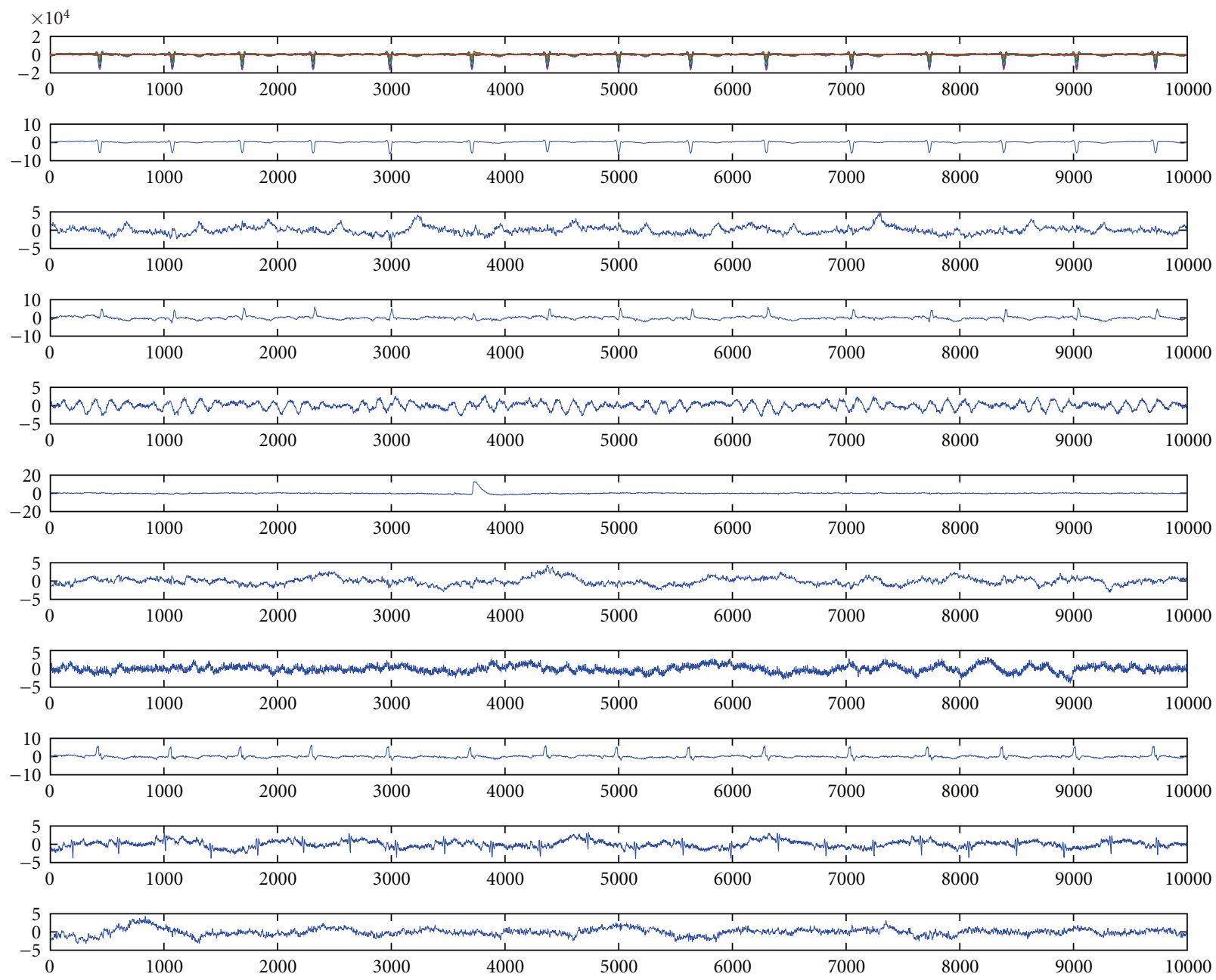

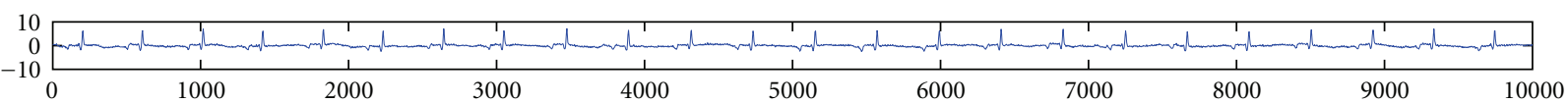

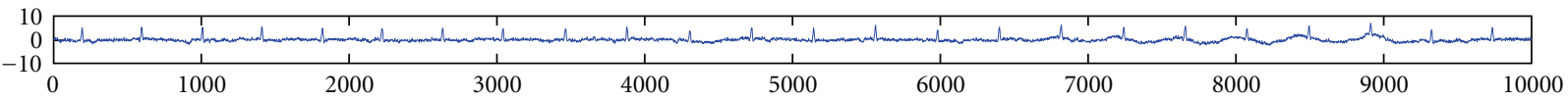

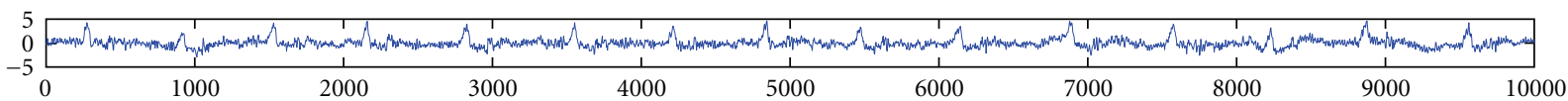

FIGURE 7: Top row: signal to be analyzed. Second to bottom row: ICA decomposition of generated signal "spontaneous activity + sinusoidal signal," where the maximum amplitude of the synthetic signal component is $500 \mathrm{fT}$.

coorbit space with respect to the frame elements (atomic decomposition), and it is also possible to reconstruct it from its sequence of moments. For the case of the $\alpha$-modulation spaces, these results can be summarized as follows.

Theorem 2. Let $1 \leq p \leq \infty, 0 \leq \alpha<1$ and $s \in \mathbb{R}$. Let $\psi \in L_{2}$ with supp $\hat{\psi}$ compact and $\hat{\psi} \in C^{2}$. Then there exists $\varepsilon_{0}>0$ with the following property. Let $\Lambda(\alpha):=\left\{\left(x_{j, k}, \omega_{j}\right)\right\}_{j, k \in \mathbb{Z}}$ denote the point set $\omega_{j}:=p_{\alpha}\left(\varepsilon_{j}\right), x_{j, k}:=\varepsilon \beta\left(\omega_{j}\right) k, 0<\varepsilon \leq \varepsilon_{0}$, where

$$
p_{\alpha}(\omega):=\operatorname{sgn}(\omega)\left((1+(1-\alpha)|\omega|)^{1 /(1-\alpha)}-1\right),
$$

then the following holds true. (i) (Atomic decomposition) Any $f \in M_{p, p}^{s, \alpha}$ can be written as

$$
f=\sum_{(j, k) \in \mathbb{Z}^{2}} c_{j, k}(f) T_{x_{j, k}} M_{\omega_{j}} D_{\beta_{\alpha}\left(\omega_{j}\right)} \psi
$$

and there exist constants $0<C_{1}, C_{2}<\infty$ (independent of $p$ ) such that

$$
\begin{aligned}
& C_{1}\|f\|_{M_{p, p}^{s, \alpha}} \\
& \leq\left(\sum_{(j, k) \in \mathbb{Z}^{2}}\left|c_{j, k}(f)\right|^{p}(1+(1-\alpha)|j|)^{((s-\alpha(1 / p-1 / 2)) /(1-\alpha)) p}\right)^{1 / p} \\
& \leq C_{2}\|f\|_{M_{p, p}^{s, \alpha} .}
\end{aligned}
$$



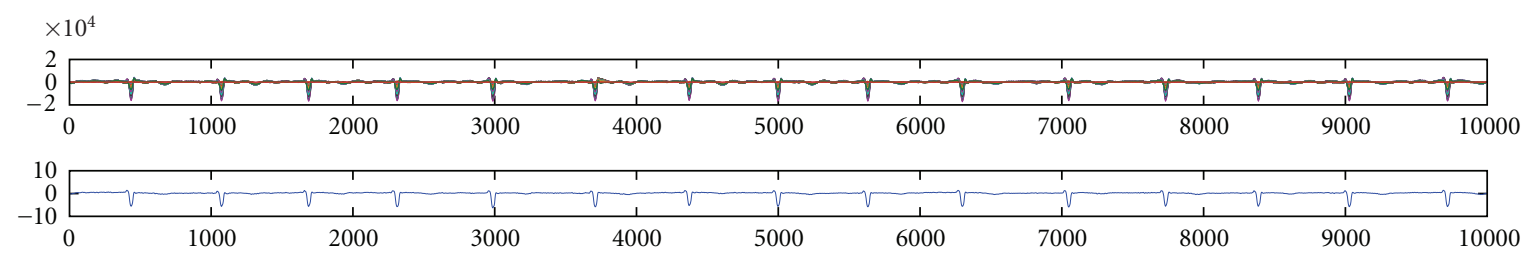

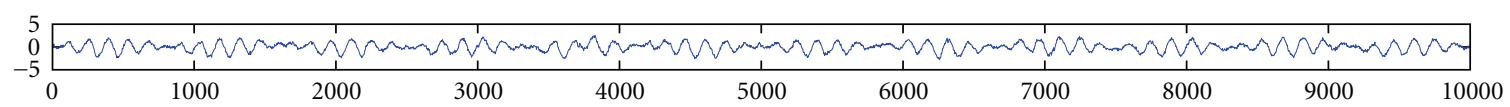

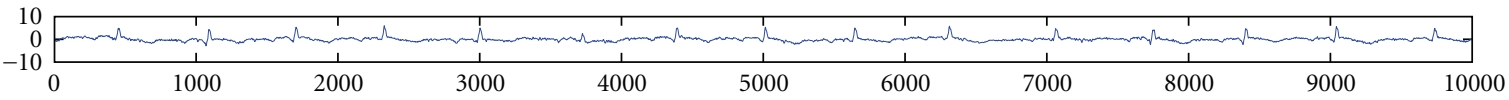

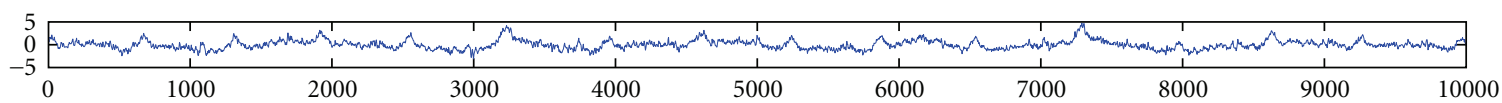
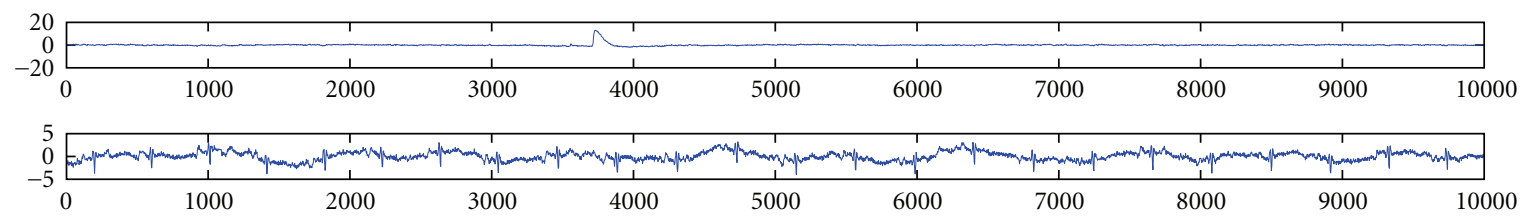

5
-5

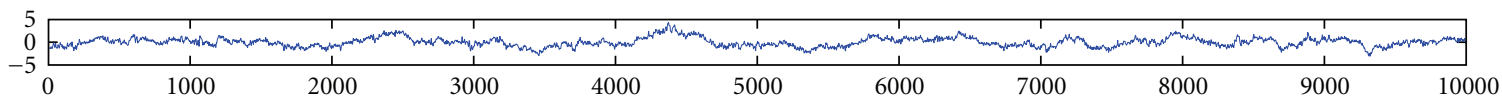

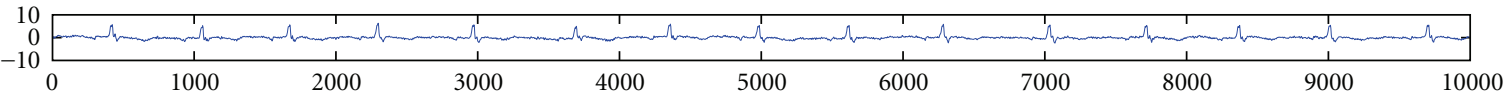

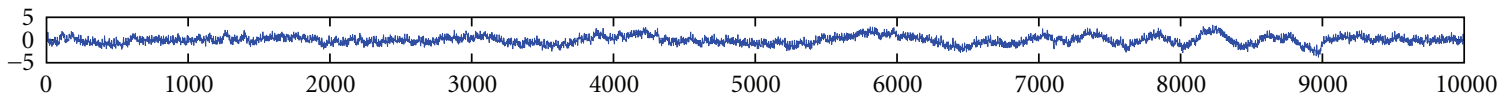

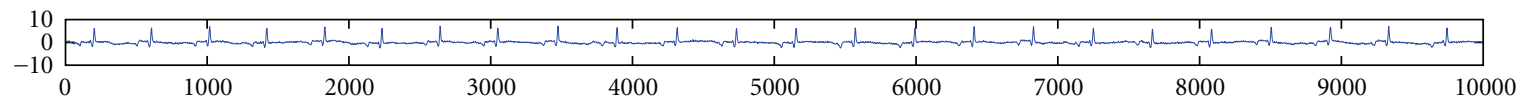

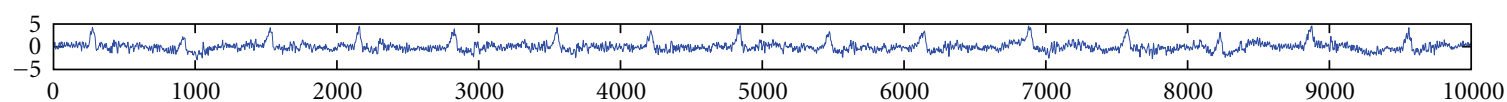

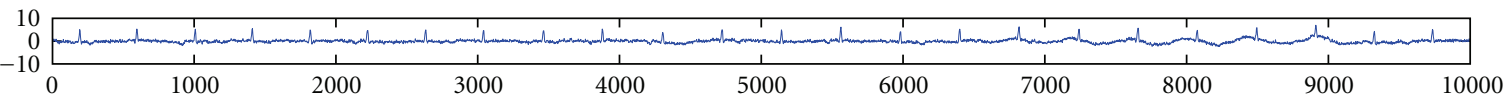

FIGURE 8: Top row: signal to be analyzed. Second to bottom row: ICA decomposition of generated signal "spontaneous activity + sinusoidal signal," where the maximum amplitude of the synthetic signal component is $1000 \mathrm{fT}$.

(ii) (Banach Frames) The set of functions $\left\{\psi_{j, k}\right\}_{j, k \in \mathbb{Z}}:=$ $\left\{T_{x_{j, k}} M_{\omega_{j}} D_{\beta_{\alpha}\left(\omega_{j}\right)} \psi\right\}_{j, k \in \mathbb{Z}^{2}}$ forms a Banach frame for $M_{p, p}^{s, \alpha}$. This means that the following hold.

(1) There exist constants $0<C_{1}, C_{2}<\infty$ (independent of p) such that

$$
\begin{aligned}
& C_{1}\|f\|_{M_{p, p}^{s, \alpha}} \\
& \leq\left(\sum_{(j, k) \in \mathbb{Z}^{2}}\left|\left\langle f, \psi_{j, k}\right\rangle\right|^{p}(1+(1-\alpha)|j|)^{((s-\alpha(1 / p-1 / 2)) /(1-\alpha)) p}\right)^{1 / p} \\
& \leq C_{2}\|f\|_{M_{p, p}^{s, \alpha} .}
\end{aligned}
$$

(2) There is a bounded, linear reconstruction operator 8 such that

$$
s\left(\left(\left\langle f, \psi_{j, k}\right\rangle_{\mathscr{H}_{1, v_{s-\alpha(1 / p-1 / 2)}} \times \mathscr{H}_{1, v_{s-\alpha(1 / p-1 / 2)}}}\right)_{j, k \in \mathbb{Z}}\right)=f .
$$

In what follows, we apply the concept of $\alpha$-modulation frames according to Theorem 2 to our multichannel data. As we have mentioned in this section, we expect that these frames provide a mixture of Gabor and wavelet frames: for small $\alpha$, the frames are similar to Gabor frames and therefore suitable for texture detection (e.g., the detection oscillatory/swinging components), whereas for $\alpha$ close to one, the frames are similar to wavelet frames and therefore suitable 

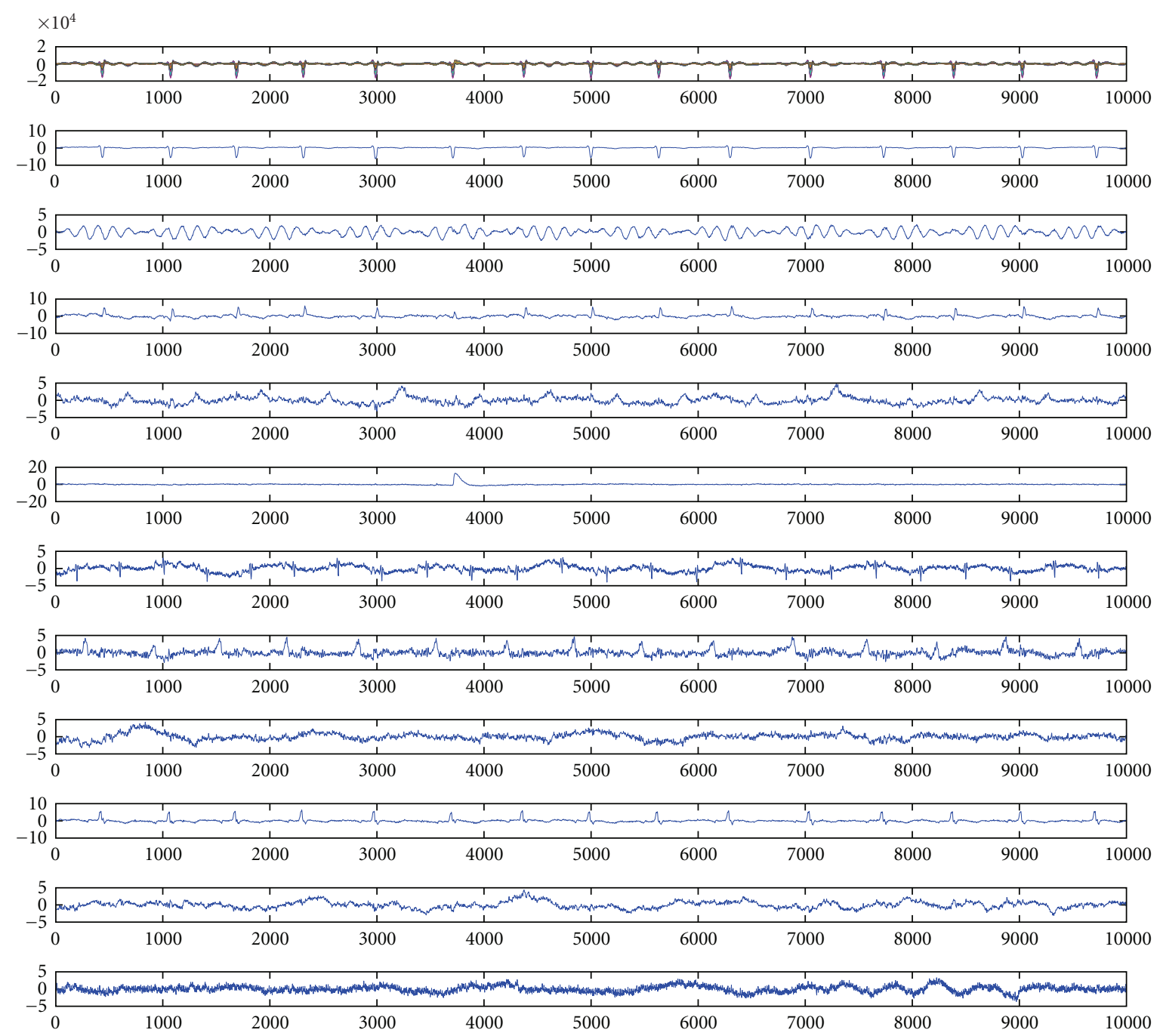

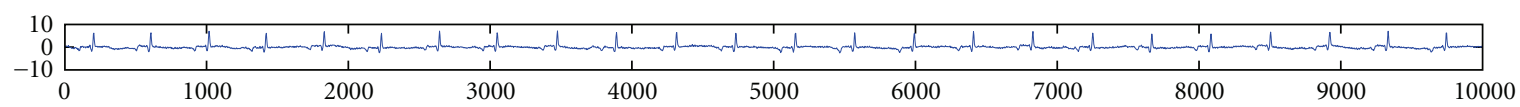

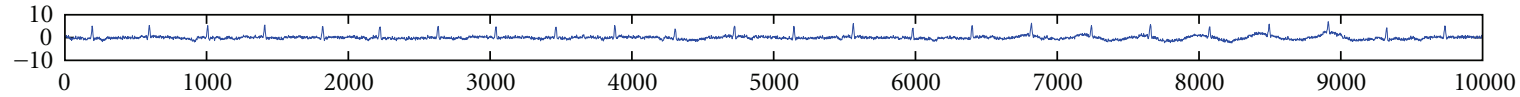

FIGURE 9: Top row: signal to be analyzed. Second to bottom row: ICA decomposition of generated signal "spontaneous activity + sinusoidal signal," where the maximum amplitude of the synthetic signal component is $2000 \mathrm{fT}$.

to extract signal components that contain singularities (e.g., rapid jumps as they appear in heart beat pattern). By varying the parameter $\alpha$, it is possible to pass from one case to the other.

\section{MULTICHANNEL DATA, $\ell_{q}$-JOINT SPARSITY AND RECOVERY MODEL}

Within this section, we focus now on multichannel data and its representation by different $\alpha$-modulation frames, the concept of joint sparsity (detection of common pattern), and finally on establishing the signal recovery model.

The aspect of common sparsity patterns was quite recently under consideration, for example, in $[21,22]$. In the framework of inverse problems/signal recovery, this issue was discussed in [23]. In the latter paper, the authors proposed an algorithm for solving vector-valued linear inverse problems with common sparsity constraints. In [24], this approach was generalized to nonlinear ill-posed inverse problems. In what follows, we revise this specific iterative thresholding scheme for solving the MCG signal recovery problem with joint sparsity constraints. We refer the interested reader to [24] in which the vector-valued joint sparsity concept is discussed and for more about the projection and thresholding techniques used therein to [25-27].

In order to cast the recovery problem as an inverse problem leading to some variational functional with a suitable sparsity constraint (forcing the detection of common 
signal pattern), we firstly have to realize that we want to act on channels of frame coefficient sequences since we aim to identify those coefficients at labels where specific medical patterns appear. To this end, we assume we are given $n$ channels containing $m$ components we wish to recover, that is, we measure data

$$
y=\left(y_{1}, \ldots, y_{n}\right) \in \bigotimes_{j=1}^{n} y=y^{n},
$$

where each channel can be represented as a sum of $m$ different components as follows:

$$
y_{j}=\sum_{i=1}^{m} f_{j}^{i}
$$

Suppose $f_{j}^{i}$ belongs, for $j=1, \ldots, n$, to some Hilbert space $\mathcal{X}_{i}$ and that each $\mathcal{X}_{i}$ is spanned by one individual $\alpha_{i}$ modulation frame $\Psi_{\alpha_{i}}=\left\{\psi_{\lambda}^{i}: \lambda \in \Lambda\left(\alpha_{i}\right)\right\}$ such that each $f_{j}^{i} \in \mathcal{X}_{i}$ can be expressed by

$$
f_{j}^{i}=\sum_{\lambda \in \Lambda\left(\alpha_{i}\right)}\left(\mathbf{f}_{j}^{i}\right)_{\lambda} \psi_{\lambda}^{i}
$$

The index $\lambda$ is a shorthand notation for $(j, k)$ and $\Lambda\left(\alpha_{i}\right)$ for the index set corresponding to the specific choice $\alpha_{i}$. This construction allows the choice of different smoothness spaces that are spanned by differently structured frames (different choice of $\alpha_{i}$ ) and involves therewith the fact that fMCG, mMCG, and MMG are of completely different nature. If we denote with $F_{i}: \mathcal{X}_{i} \rightarrow \ell_{2}\left(\Lambda_{\alpha_{i}}\right)$ the associated $\alpha_{i}$ modulation analysis operator, compare with (11), and with $\mathrm{id}_{i}: X_{i} \rightarrow \mathcal{y}$ the embedding operator, we may define the relationship between the data of the $j$ th channel $y_{j}$ and the frame coefficients $\mathbf{f}_{j}=\left(\mathbf{f}_{j}^{1}, \ldots, \mathbf{f}_{j}^{m}\right)$ of the $m$ associated components

$$
y_{j}=A \mathbf{f}_{j}=A\left(\mathbf{f}_{j}^{1}, \ldots, \mathbf{f}_{j}^{m}\right)=\sum_{i=1}^{m} \mathrm{id}_{i} F_{i}^{*} \mathbf{f}_{j}^{i},
$$

where $\mathbf{f}_{j}^{i} \in \ell_{2}\left(\Lambda\left(\alpha_{i}\right)\right)$, that is, $\mathbf{f}_{j}=\left(\mathbf{f}_{j}^{1}, \ldots, \mathbf{f}_{j}^{m}\right) \in$ $\otimes_{i=1}^{m} \ell_{2}\left(\Lambda_{\alpha_{i}}\right)$. Consequently,

$$
\begin{gathered}
A: \bigotimes_{i=1}^{m} \ell_{2}\left(\Lambda_{\alpha_{i}}\right) \longrightarrow y \quad \text { via }\left(\mathbf{f}^{1}, \ldots, \mathbf{f}^{m}\right) \longmapsto \sum_{i=1}^{m} \mathrm{id}_{i} F_{i}^{*} \mathbf{f}^{i}, \\
A^{*}: y \longrightarrow \bigotimes_{i=1}^{m} \ell_{2}\left(\Lambda_{\alpha_{i}}\right) \quad \text { via } y \longmapsto\left(F_{1} \mathrm{id}_{1}^{*} y, \ldots, F_{m} \mathrm{id}_{m}^{*} y\right) .
\end{gathered}
$$

Following the arguments in $[21,23]$ on joint sparsity and denoting with $\mathbf{f}^{i}=\left(\mathbf{f}_{1}^{i}, \ldots, \mathbf{f}_{n}^{i}\right)$ the vector of frame coefficient sequences of all $n$ channels with respect to one specific signal component, a reasonable measure that forces a coupling of nonvanishing frame coefficients through all $n$ channels (representing a common morphology) is of the form

$$
\Phi\left(\mathbf{f}^{i}\right)=\Phi_{p_{i}, q_{i}, \omega^{i}}\left(\mathbf{f}^{i}\right)=\sum_{\lambda \in \Lambda\left(\alpha_{i}\right)} \omega_{\lambda}^{i}\left\|\left(\mathbf{f}_{.}^{i}\right)_{\lambda}\right\|_{q_{i}}^{p_{i}}
$$

with $q_{i} \in[1, \infty], p_{i} \in\left\{1, q_{i}\right\}, \omega_{\lambda}^{i} \geq c>0$, where the $q_{i^{-}}$ norm is taken with respect to the channel index, that is,

$$
\left\|\left(\mathbf{f}^{i}\right)_{\lambda}\right\|_{q_{i}}=\left(\sum_{j=1}^{n}\left|\left(\mathbf{f}_{j}^{i}\right)_{\lambda}\right|^{q_{i}}\right)^{1 / q_{i}} .
$$

Forcing for a common sparsity pattern (e.g., common heart beats), a coupling of the different channels is advantageous and can be achieved when setting, for example, $q_{i}=2$ and $p_{i}=1$.

Summarizing the findings, an $m$ component signal recovery model in a variational formulation reads as

$$
\begin{aligned}
J_{\mu, p, q}(\mathbf{f}) & =J_{\mu, p, q}\left(\mathbf{f}^{1}, \ldots, \mathbf{f}^{m}\right) \\
& =\sum_{j=1}^{n}\left\|y_{j}-A \mathbf{f}_{j}\right\|_{y}^{2}+2 \sum_{i=1}^{m} \mu_{i} \Phi_{p_{i}, q_{i}, \omega^{i}}\left(\mathbf{f}^{i}\right),
\end{aligned}
$$

or in compact form

$$
J_{\mu, p, q}(\mathbf{f})=\|y-\tilde{A} \mathbf{f}\|_{y^{n}}^{2}+2 \sum_{i=1}^{m} \mu_{i} \Phi_{p_{i}, q_{i}, \omega^{i}}\left(\mathbf{f}^{i}\right),
$$

where we have defined the following shorthand notations:

$$
\begin{gathered}
\tilde{A} y=\left(A y_{1}, \ldots, A y_{n}\right), \quad \mu=\left(\mu_{1}, \ldots, \mu_{m}\right), \\
p=\left(p_{1}, \ldots, p_{m}\right), \quad q=\left(q_{1}, \ldots, q_{m}\right) .
\end{gathered}
$$

An approximation to the original $m$ different signal components (mMCG, fMCG, MMG, etc. ) is now computed by means of the minimizer $\mathbf{f} \in\left(\bigotimes_{i=1}^{m} \ell_{2}\left(\Lambda_{\alpha_{i}}\right)\right)^{n}$ of (26). Unfortunately, a direct approach toward its minimization leads to a nonlinear optimality system where the frame coefficients are coupled. Instead, we propose to replace (26) by a sequence of functionals that are much easier to minimize and for which the sequence of the corresponding minimizers converges at least to a critical point of (26). To be explicit, for $\mathbf{f} \in\left(\otimes_{i=1}^{m} \ell_{2}\left(\Lambda_{\alpha_{i}}\right)\right)^{n}$ and some auxiliary $\mathbf{a} \in\left(\otimes_{i=1}^{m} \ell_{2}\left(\Lambda_{\alpha_{i}}\right)\right)^{n}$, we define a surrogate functional

$J_{\mu, p, q}^{s}(\mathbf{f}, \mathbf{a}):=J_{\mu, p, q}(\mathbf{f})+C\|\mathbf{f}-\mathbf{a}\|_{\left(\bigotimes_{i=1}^{m} \ell_{2}\left(\Lambda_{\alpha_{i}}\right)\right)^{n}}^{2}-\|\tilde{A} \mathbf{f}-\tilde{A} \mathbf{a}\|_{y^{n},}^{2}$

and create an iteration process by

(1) picking some initial guess $[\mathbf{f}]_{0} \in\left(\bigotimes_{i=1}^{m} \ell_{2}\left(\Lambda_{\alpha_{i}}\right)\right)^{n}$ and some proper constant $C>0$;

(2) deriving a sequence $\left([\mathbf{f}]_{k}\right), k=0,1, \ldots$, by the iteration

$$
[\mathbf{f}]_{k+1}=\arg \min _{\mathbf{f} \in\left(\bigotimes_{i=1}^{m} \ell_{2}\left(\Lambda_{\alpha_{i}}\right)\right)^{n}} J_{\mu, p, q}^{s}\left(\mathbf{f},[\mathbf{f}]_{k}\right), \quad k=0,1,2, \ldots
$$

It will turn out that the minimizers of the surrogate functionals are easily computed. In particular, the problem decouples, and every frame coefficient can be treated separately. In order to ensure the existence of global minimizers, norm convergence of the iterates $[\mathbf{f}]_{k}$, and regularization properties, some weak assumptions (exhibiting no significant restriction) have to be made, see for details $[24,28]$ and references therein. 


\section{ALGORITHMIC IMPLEMENTATION AND NUMERICAL EXPERIMENTS}

In order to specify the numerical algorithm, we have to set up the constant $C$ and to derive the necessary conditions for a minimum of $J_{\mu, p, q}^{s}(\mathbf{f}, \mathbf{a})$, yielding the concrete proceeding of iteration (30).

The constant $C$ can be easily determined, see [28]. For $\mathbf{f} \in\left(\otimes_{i=1}^{m} \ell_{2}\left(\Lambda_{\alpha_{i}}\right)\right)^{n}$, we have

$$
\langle\widetilde{A} \mathbf{f}, \tilde{A} \mathbf{f}\rangle_{y^{n}}=\sum_{j=1}^{n}\left\|A \mathbf{f}_{j}\right\|_{y}^{2} .
$$

Since $A$ is bounded, it holds $\|A\|=\left\|A^{*}\right\|$, and we may estimate

$$
\begin{aligned}
\left\langle A^{*} y, A^{*} y\right\rangle_{\bigotimes_{i=1}^{m} \ell_{2}\left(\Lambda_{\alpha_{i}}\right)} & =\sum_{i=1}^{m}\left\|F_{i} \mathrm{id}_{i}^{*} y\right\|_{\ell_{2}\left(\Lambda_{\alpha_{i}}\right)}^{2} \\
& \leq \sum_{i=1}^{m}\left\|F_{i}\right\|^{2}\left\|\mathrm{id}_{i}^{*}\right\|^{2}\|y\|_{y}^{2} .
\end{aligned}
$$

Therefore,

$$
\begin{aligned}
\|\tilde{A} \mathbf{f}\|^{2} & \leq \sum_{j=1}^{n} \sum_{i=1}^{m}\left\|F_{i}\right\|^{2}\left\|\mathrm{id}_{i}^{*}\right\| \mathbf{f}_{j} \|_{\bigotimes_{i=1}^{m} \ell_{2}\left(\Lambda_{\alpha_{i}}\right)}^{2} \\
& \leq \sum_{i=1}^{m}\left\|F_{i}\right\|^{2}\left\|\mathrm{id}_{i}^{*}\right\|^{2}\|\mathbf{f}\|_{y^{n}}^{2},
\end{aligned}
$$

and consequently, $C$ must be chosen such that $\|\tilde{A}\|^{2} \leq$ $\sum_{i=1}^{m}\left\|F_{i}\right\|^{2}\left\|\mathrm{id}_{i}^{*}\right\|^{2}<C$. In order to specify the algorithm, we firstly rewrite (29) as

$$
\begin{aligned}
J_{\mu, p, q}^{s}(\mathbf{f}, \mathbf{a})= & \left\|C^{-1} \tilde{A}^{*} y+\mathbf{a}-C^{-1} \tilde{A}^{*} \tilde{A} \mathbf{a}-\mathbf{f}\right\|_{\left(\bigotimes_{i=1}^{m} \ell_{2}\left(\Lambda_{\alpha_{i}}\right)\right)^{n}}^{2} \\
& +\frac{2}{C} \sum_{i=1}^{m} \mu_{i} \Phi_{p_{i}, q_{i}, \omega_{i}}\left(\mathbf{f}^{i}\right)+\text { rest }
\end{aligned}
$$

where the "rest" does not depend on $\mathbf{f}$. The right-hand side without the "rest" can be rewritten as follows:

$$
\begin{aligned}
J_{\mu, p, q}^{s}(\mathbf{f}, \mathbf{a}) & - \text { rest } \\
= & \sum_{j=1}^{n}\left\|C^{-1} A^{*} y_{j}+\mathbf{a}_{j}-C^{-1} A^{*} A \mathbf{a}_{j}-\mathbf{f}_{j}\right\|_{\otimes_{i=1}^{m} \ell_{2}\left(\Lambda_{\alpha_{i}}\right)}^{2} \\
& +\frac{2}{C} \sum_{i=1}^{m} \mu_{i} \Phi_{p_{i}, q_{i}, \omega_{i}}\left(\mathbf{f}^{i}\right)
\end{aligned}
$$

$$
\begin{aligned}
= & \sum_{j=1}^{n} \sum_{i=1}^{m}\left\|C^{-1} F_{i} \mathrm{id}_{i}^{*}\left(y_{j}-A \mathbf{a}_{j}\right)+\mathbf{a}_{j}^{i}-\mathbf{f}_{j}^{i}\right\|_{\ell_{2}\left(\Lambda_{\alpha_{i}}\right)}^{2} \\
& +\frac{2}{C} \sum_{i=1}^{m} \mu_{i} \Phi_{p_{i}, q_{i}, \omega_{i}}\left(\mathbf{f}^{i}\right) \\
= & \sum_{i=1}^{m}\left\{\sum_{j=1}^{n}\left\|C^{-1} F_{i} \mathrm{id}_{i}^{*}\left(y_{j}-A \mathbf{a}_{j}\right)+\mathbf{a}_{j}^{i}-\mathbf{f}_{j}^{i}\right\|_{\ell_{2}\left(\Lambda_{\alpha_{i}}\right)}^{2}\right. \\
& \left.+\frac{2 \mu_{i}}{C} \Phi_{p_{i}, q_{i}, \omega_{i}}\left(\mathbf{f}^{i}\right)\right\} \\
= & \sum_{i=1}^{m} \sum_{\lambda \in \Lambda\left(\alpha_{i}\right)}\left\{\sum_{j=1}^{n}\left|\left(C^{-1} F_{i} \mathrm{id}_{i}^{*}\left(y_{j}-A \mathbf{a}_{j}\right)+\mathbf{a}_{j}^{i}-\mathbf{f}_{j}^{i}\right)_{\lambda}\right|^{2}\right. \\
& \left.+\frac{2 \mu_{i}}{C} \omega_{\lambda}^{i}\left\|\left(\mathbf{f}_{.}^{i}\right)_{\lambda}\right\|_{q_{i}}^{p_{i}}\right\} \\
= & \sum_{i=1}^{m} \sum_{\lambda \in \Lambda\left(\alpha_{i}\right)}\left\{\left\|\left(C^{-1} F_{i} \mathrm{id}_{i}^{*}(y .-A \mathbf{a} .)+\mathbf{a}_{.}^{i}\right)_{\lambda}-\left(\mathbf{f}_{.}^{i}\right)_{\lambda}\right\|_{2}^{2}\right. \\
& \left.+\frac{2 \mu_{i}}{C} \omega_{\lambda}^{i}\left\|\left(\mathbf{f}_{.}^{i}\right)_{\lambda}\right\|_{q_{i}}^{p_{i}}\right\} .
\end{aligned}
$$

For $p_{i}=q_{i}$, the variational equations completely decouple, and a straightforward minimization with respect to $\left(\mathbf{f}_{j}^{i}\right)_{\lambda}$ yields the necessary conditions. For $p_{i}=1$, the term within the brackets is of the following general structure:

$$
\|y-x\|_{2}^{2}+v\|x\|_{q}
$$

with $x, y \in \mathbb{R}^{n}$ and some $v \in \mathbb{R}_{+}$. The minimizing element $x^{*}$ of this functional is easily obtained, see $[23,24]$,

$$
x^{*}=\left(I-P_{B_{q^{\prime}}(v)}\right)(y)
$$

where $P_{B_{q^{\prime}}(\nu)}$ is the orthogonal projection onto the ball $B_{q^{\prime}}(\nu)$ with radius $\nu$ in the dual norm of $\|\cdot\|_{q}$ (i.e., $1 / q+1 / q^{\prime}=$ $1)$. In general, the evaluation of $P_{B_{q^{\prime}}(v)}$ is rather difficult and only for a few individual choices of $q$ given, see $[23,28]$. For the case $q_{i}=2$ (on which we will focus), the projection is explicitly given by

$$
P_{B_{q^{\prime}}(v)}(y)= \begin{cases}y, & \text { if }\|y\|_{2} \leq \nu, \\ \nu \frac{y}{\|y\|_{2}}, & \text { otherwise. }\end{cases}
$$

In what follows, we adapt now the algorithm to our concrete medical signal analysis problem. The 151-channel SQUID data consist (beside biological background noise) essentially of four components: $\mathrm{MCG}, \mathrm{mMCG}, \mathrm{MMG}$, and "motion artifacts." We aim to split the multichannel signal into fMCG + mMCG and MMG + "motion artifacts." Therefore, we set $n=151$ and $m=2$. Since the fMCG + mMCG is assumed to be coupled through all the 151 channels, we put on this signal component $(i=1)$ the joint sparsity constraint. This ensures the natural condition that heart beat patterns appear in all the channels at the same 
(temporal) location. On the other hand, since the MMG + "motion artifacts" component $(i=2)$ can be arbitrarily (but sparsely) localized, we do not put a common sparsity constraint on this signal component. These constraints setup can be realized when choosing $p_{1}=1, q_{1}=2$, and $p_{2}=$ $q_{2}=1$. Finally, we have to select adequate $\alpha_{i}$-modulation frames. Since the $\mathrm{AMCG}+\mathrm{mMCG}$ component is allowed to consist of rapid jumps (being close to singularities), we prefer $\alpha_{1}$ close to one. In contrast, the MMG + "motion artifacts" component is supposed to be much smoother, we prefer $\alpha_{2}$ close to zero. For this particular situation, the variational functional reads as

$$
\begin{aligned}
& J_{\left(\mu_{1}, \mu_{2}\right),(1,1),(2,1)}^{s}(\mathbf{f}, \mathbf{a})-\text { rest } \\
&=\sum_{\lambda \in \Lambda\left(\alpha_{i}\right)}\{\left\|\left(C^{-1} F_{1} \mathrm{id}_{1}^{*}(y \cdot-A \mathbf{a} .)+\mathbf{a}^{1}\right)_{\lambda}-\left(\mathbf{f}^{1}\right)_{\lambda}\right\|_{2}^{2} \\
&+\frac{2 \mu_{1}}{C} \omega_{\lambda}^{1}\left\|\left(\mathbf{f}^{1}\right)_{\lambda}\right\|_{2} \\
&+\left\|\left(C^{-1} F_{2} \mathrm{id}_{2}^{*}(y \cdot-A \mathbf{a} .)+\mathbf{a}^{2}\right)_{\lambda}-\left(\mathbf{f}_{.}^{2}\right)_{\lambda}\right\|_{2}^{2} \\
&\left.+\frac{2 \mu_{2}}{C} \omega_{\lambda}^{2}\left\|\left(\mathbf{f}^{2}\right)_{\lambda}\right\|_{1}\right\} .
\end{aligned}
$$

Defining

$$
M^{i}\left(y_{j}, \mathbf{a}_{j}\right):=C^{-1} F_{i} \mathrm{id}_{i}^{*}\left(y_{j}-A \mathbf{a}_{j}\right)+\mathbf{a}_{j}^{i},
$$

the individual $\alpha_{1}$-modulation frame coefficients of signal component 1 are given thanks to (37) and (38) by

$$
\begin{aligned}
\left(\mathbf{f}^{1}\right)_{\lambda}= & \left(\left(\mathbf{f}_{1}^{1}\right)_{\lambda}, \ldots,\left(\mathbf{f}_{151}^{1}\right)_{\lambda}\right) \\
= & \left(I-P_{B_{2}\left(\mu_{1} \omega_{\lambda}^{1} / C\right)}\right) \\
& \times\left(\left(M^{1}\left(y_{1}, \mathbf{a}_{1}\right)\right)_{\lambda}, \ldots,\left(M^{1}\left(y_{151}, \mathbf{a}_{151}\right)\right)_{\lambda}\right),
\end{aligned}
$$

for all $\lambda \in \Lambda\left(\alpha_{1}\right)$, whereas the $\alpha_{2}$-modulation frame coefficients of signal component 2 are given by

$$
\begin{aligned}
\left(\mathbf{f}^{2}\right)_{\lambda} & =\left(\left(\mathbf{f}_{1}^{2}\right)_{\lambda}, \ldots,\left(\mathbf{f}_{151}^{2}\right)_{\lambda}\right) \\
& =S_{\mu_{2} \omega_{\lambda}^{2} / C}\left(\left(M^{2}\left(y_{1}, \mathbf{a}_{1}\right)\right)_{\lambda}, \ldots,\left(M^{2}\left(y_{151}, \mathbf{a}_{151}\right)\right)_{\lambda}\right),
\end{aligned}
$$

for all $\lambda \in \Lambda\left(\alpha_{2}\right)$ and where $S_{\mu_{2} \omega_{\lambda}^{2} / C}$ denotes the well-known nonlinear soft-shrinkage operator (acting on each channel individually).

With the help of (41) and (42), the iteration (30) can finally be written as

$$
\begin{aligned}
& {\left[\begin{array}{c}
\left(\mathbf{f}^{1}\right)_{\lambda} \\
\left(\mathbf{f}^{2}\right)_{\lambda}
\end{array}\right]_{k+1}} \\
& =\left(\begin{array}{c}
\left.\left(I-P_{B_{2}\left(\mu_{1} \omega_{\lambda}^{1} / C\right)}\right)\left(\left(M^{1}\left(y_{1},\left[\mathbf{f}_{1}\right]_{k}\right)\right)_{\lambda}, \ldots,\left(M^{1}\left(y_{151},\left[\mathbf{f}_{151}\right]_{k}\right)\right)_{\lambda}\right)\right) \\
S_{\mu_{2} \omega_{\lambda}^{2} / C}\left(\left(M^{2}\left(y_{1},\left[\mathbf{f}_{1}\right]_{k}\right)\right)_{\lambda}, \ldots,\left(M^{2}\left(y_{151},\left[\mathbf{f}_{151}\right]_{k}\right)\right)_{\lambda}\right)
\end{array}\right) .
\end{aligned}
$$

Procedure (43) is now applied to the SQUID multichannel data and compared with the ICA-based algorithm JADE.
The data we aim to analyze are for reasons of verification synthetically generated and consist of two components. One component is a measured spontaneous activity (fMCG and mMCG, i.e., fetal and maternal heart beats filtered with a high-pass filter at $0.5 \mathrm{~Hz}$ ), see for a few individual channels Figure 3. The second component is a combination of two sinusoidal functions $(7 \mathrm{~Hz}$ and $0.6 \mathrm{~Hz}$, which should resemble a growing and vanishing uterine contraction, i.e., motion artifacts + possible MCG), see for a few individual channels Figure 4. The sinusoidal signal has its maximum amplitude at a channel in the center of the SQUID array whereas the amplitudes of the other sensors were attenuated by a Gaussian weight function, see Figure 2. The sum of the two components (spontaneous activity + sinusoidal signal) forms the data basis to be analyzed. In order to evaluate advantages and/or disadvantages of the two methods, the maximum amplitude at the center of appearance of the synthetic data component was gradually decreased from $2000 \mathrm{fT}$ to $125 \mathrm{fT}(2000 \mathrm{fT}, 1000 \mathrm{fT}, 500 \mathrm{fT}, 250 \mathrm{fT}$, and $125 \mathrm{fT})$.

For the sake of simple illustration, we have restricted the visualization of data and reconstruction/decomposition results to one channel (JADE algorithm) and two channels (our proposed algorithm). The results of the JADE algorithm are visualized in Figures 5, 6, 7, 8, and 9 (for one particular channel). As usual for an ICA analysis, numerous components (here 12) are derived. Clearly, it is visible in the figures that even the fetal and maternal heart beats are completely decomposed. As a quantitative observation, in Figure 5, a sinusoidal structure is not reconstructed at all. In Figure 6, a very noisy version of the sinusoidal structure could be separated (see 5 th row). In the remaining Figures ( 7 (5th row), 8 (3rd row), and 9 (3rd row)), the sinusoidal structure could be sufficiently reconstructed.

The results that we have obtained with the application of our proposed iteration scheme (43) (setting $\alpha_{1}=0.9, \alpha_{2}=$ 0 , and $\mu_{1}=\mu_{2}=0.001$ ) are visualized in Figure 10 (sinusoidal signal with maximum $125 \mathrm{fT}$ ), 11 (sinusoidal signal with maximum $250 \mathrm{fT}$ ), 12 (sinusoidal signal with maximum $500 \mathrm{fT}$ ), 13 (sinusoidal signal with maximum $1000 \mathrm{fT}$ ), and 14 (sinusoidal signal with maximum $2000 \mathrm{fT}$ ). In order to show the reconstruction results also for different channels, we have switched the visualization of the channels. In particular, we have shown the reconstruction/decomposition results for channels 80 and 40 in Figure 10, for channels 20 and 40 in Figures 11-13, and for channels 40 and 41 in Figure 14.

Summarizing the numerical results, we may deduce that in comparison with the JADE algorithm, our proposed algorithm recovers all simulated sinusoidal signal structures (containing no noise contribution as it is the case for JADE reconstructions). For the critical data examples (with $125 \mathrm{fT}, 250 \mathrm{fT}$, and $500 \mathrm{fT}$ maximum amplitude) in which the sinusoidal signal component was very weak, the recovered signal contains for the maximum amplitude of $125 \mathrm{fT}$ at least cyclic-modulated oscillations (not fitting well with the shape of the originally generated synthetic signal), and for $250 \mathrm{fT}$ and $500 \mathrm{fT}$ gradually improved recovery results. This is comparable to the results achieved by the JADE algorithm. 

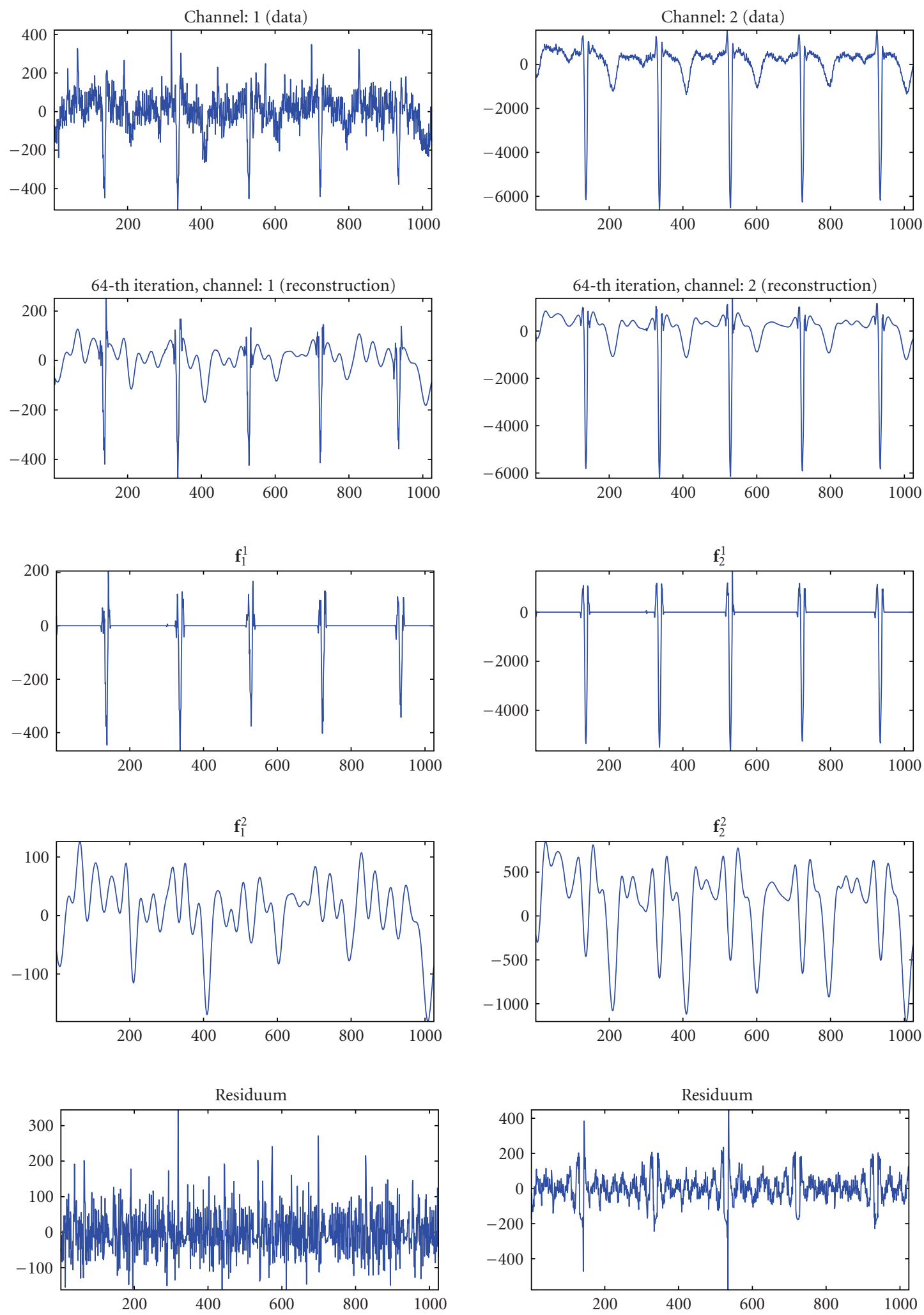

(a)

(b)

FIgURE 10: The reconstruction/decomposition of channels 80 (left) and 40 (right). Top row: data to be analyzed (spontaneous activity + sinusoidal signal with maximum $125 \mathrm{fT}$ ). Second row: reconstructions $\mathbf{f}_{1}^{1}+\mathbf{f}_{1}^{2}$ (left) and $\mathbf{f}_{2}^{1}+\mathbf{f}_{2}^{2}$ (right). Third row: fMCG + mMCG reconstructed component $\mathbf{f}_{1}^{1}$ (left) and $\mathbf{f}_{2}^{1}$ (right). Fourth row: MMG + "motion artifacts" reconstructed component $\mathbf{f}_{1}^{2}$ (left) and $\mathbf{f}_{2}^{2}$ (right). Bottom row: residuum (containing noise, contribution of maternal (minor) and partially fetal heart beat components, and background signals). 

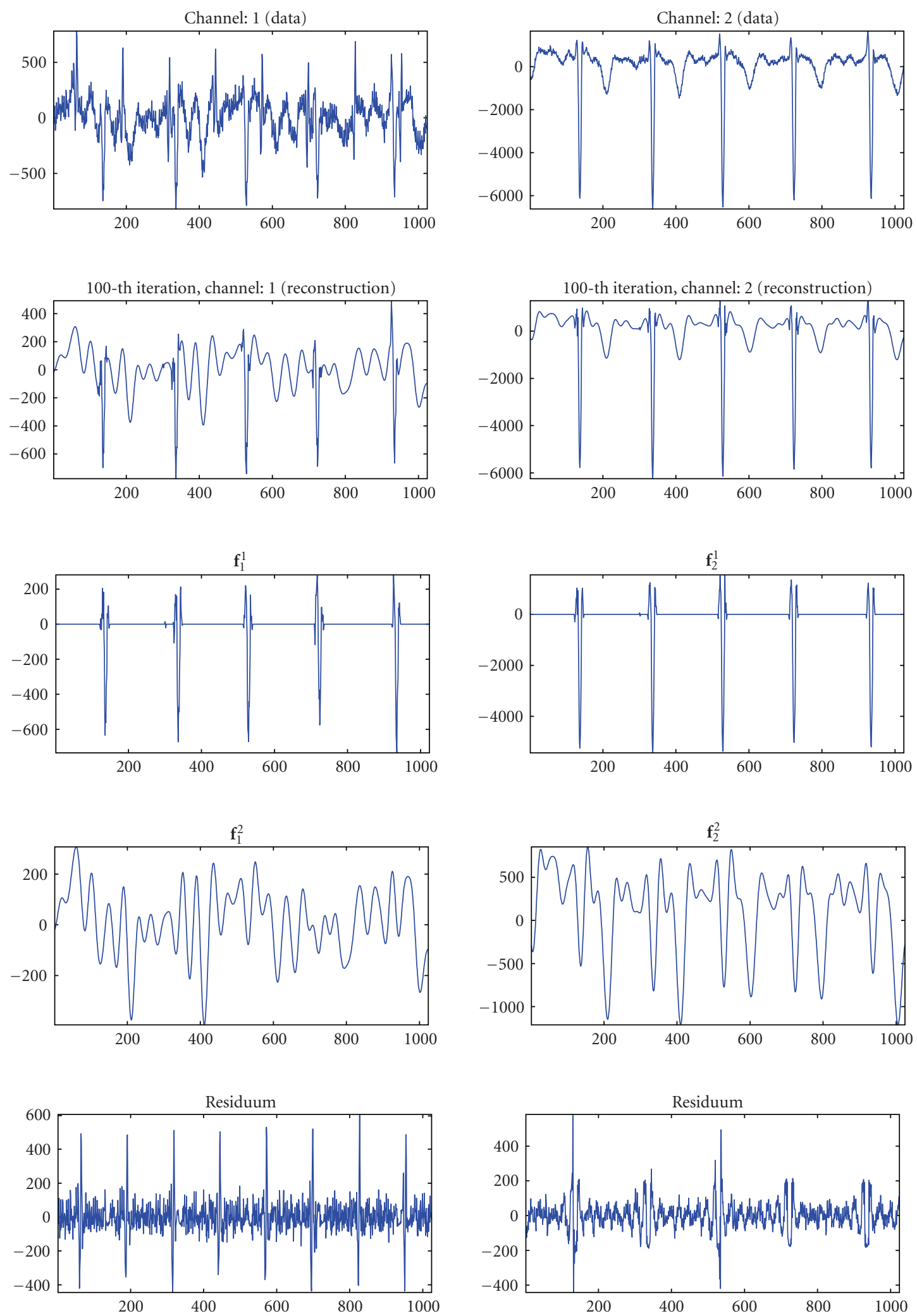

(a)

(b)

FIGURE 11: The reconstruction/decomposition of channels 20 (left) and 40 (right). Top row: data to be analyzed (spontaneous activity + sinusoidal signal with maximum $250 \mathrm{fT}$ ). Second row: reconstructions $\mathbf{f}_{1}^{1}+\mathbf{f}_{1}^{2}$ (left) and $\mathbf{f}_{2}^{1}+\mathbf{f}_{2}^{2}$ (right). Third row: fMCG $+\mathrm{mMCG}$ reconstructed component $\mathbf{f}_{1}^{1}$ (left) and $\mathbf{f}_{2}^{1}$ (right). Fourth row: MMG + "motion artifacts" reconstructed component $\mathbf{f}_{1}^{2}$ (left) and $\mathbf{f}_{2}^{2}$ (right). Bottom row: residuum (containing noise, contribution of maternal (minor) and fetal heart beat components, and background signals). 

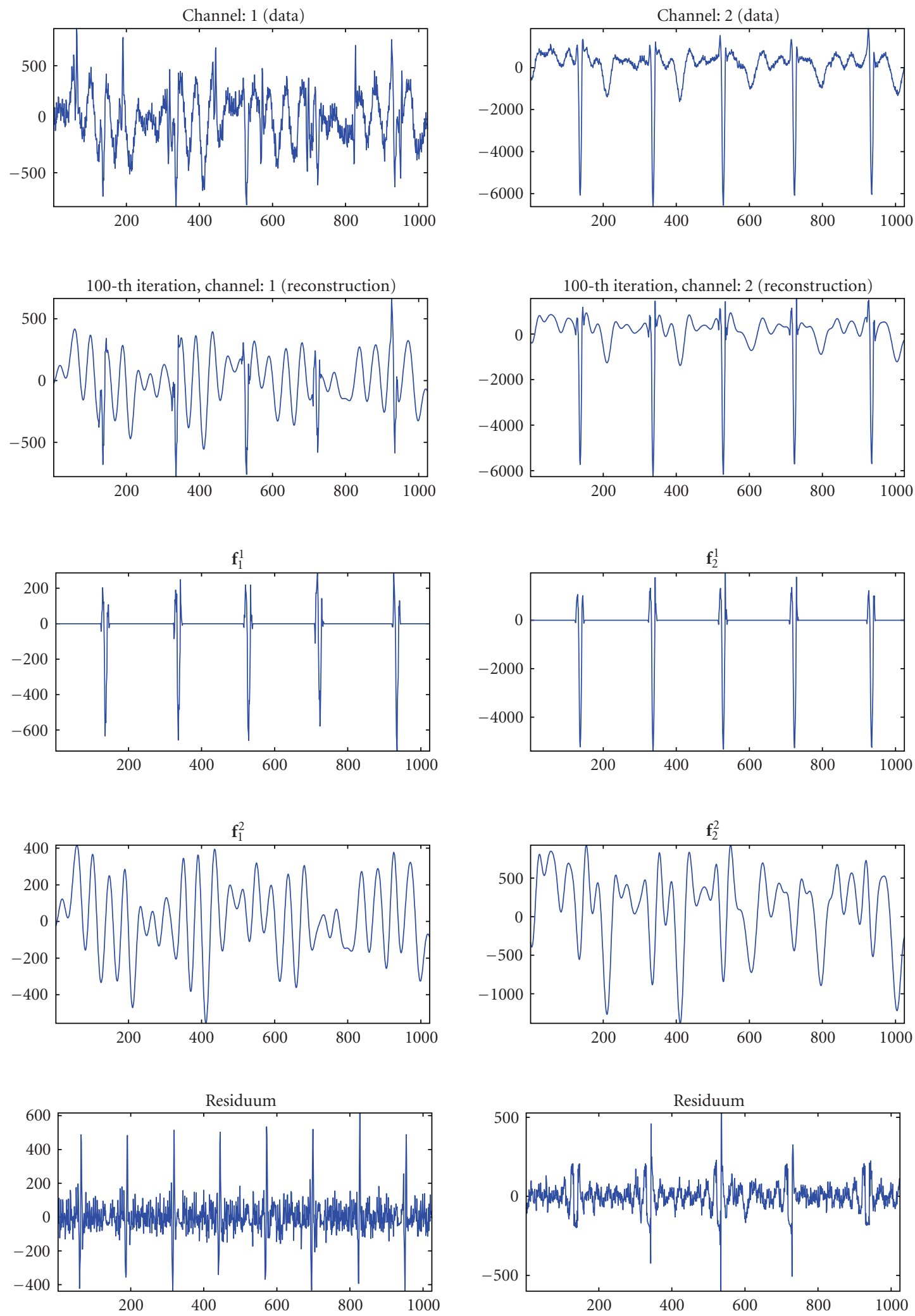

(a)

(b)

FIGURE 12: The reconstruction/decomposition of channels 20 (left) and 40 (right). Top row: data to be analyzed (spontaneous activity + sinusoidal signal with maximum $500 \mathrm{fT}$ ). Second row: reconstructions $\mathbf{f}_{1}^{1}+\mathbf{f}_{1}^{2}$ (left) and $\mathbf{f}_{2}^{1}+\mathbf{f}_{2}^{2}$ (right). Third row: fMCG $+\mathrm{mMCG}$ reconstructed component $\mathbf{f}_{1}^{1}$ (left) and $\mathbf{f}_{2}^{1}$ (right). Fourth row: MMG + "motion artifacts" reconstructed component $\mathbf{f}_{1}^{2}$ (left) and $\mathbf{f}_{2}^{2}$ (right). Bottom row: residuum (containing noise, contribution of maternal (minor) and fetal heart beat components, and background signals). 

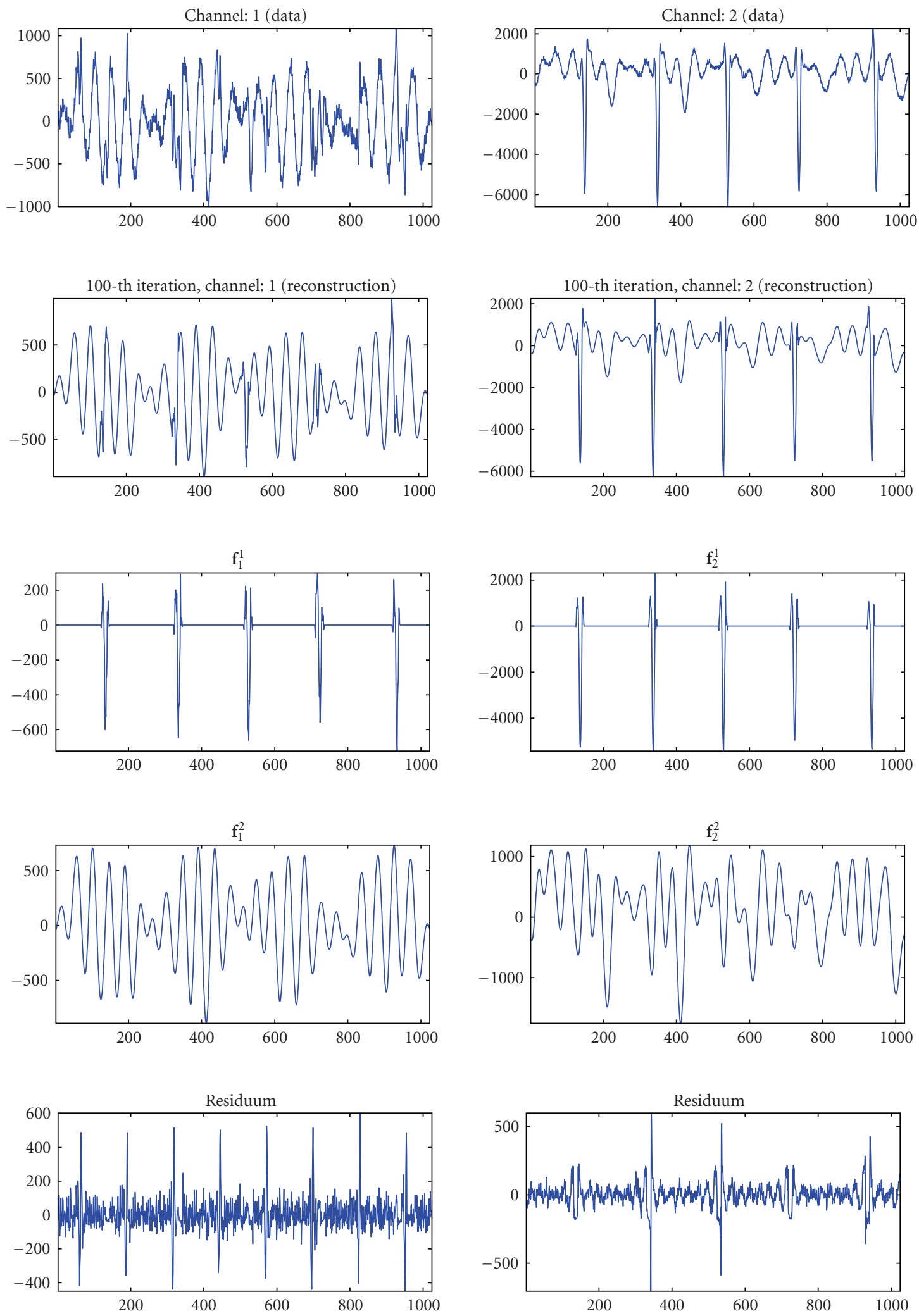

(a)

(b)

FIGURE 13: The reconstruction/decomposition of channels 20 (left) and 40 (right). Top row: data to be analyzed (spontaneous activity + sinusoidal signal with maximum $1000 \mathrm{fT}$ ). Second row: reconstructions $\mathbf{f}_{1}^{1}+\mathbf{f}_{1}^{2}$ (left) and $\mathbf{f}_{2}^{1}+\mathbf{f}_{2}^{2}$ (right). Third row: $\mathrm{fMCG}+\mathrm{mMCG}$ reconstructed component $\mathbf{f}_{1}^{1}$ (left) and $\mathbf{f}_{2}^{1}$ (right). Fourth row: MMG + "motion artifacts" reconstructed component $\mathbf{f}_{1}^{2}$ (left) and $\mathbf{f}_{2}^{2}$ (right). Bottom row: residuum (containing noise, contribution of maternal (minor) and fetal heart beat components, and background signals). 

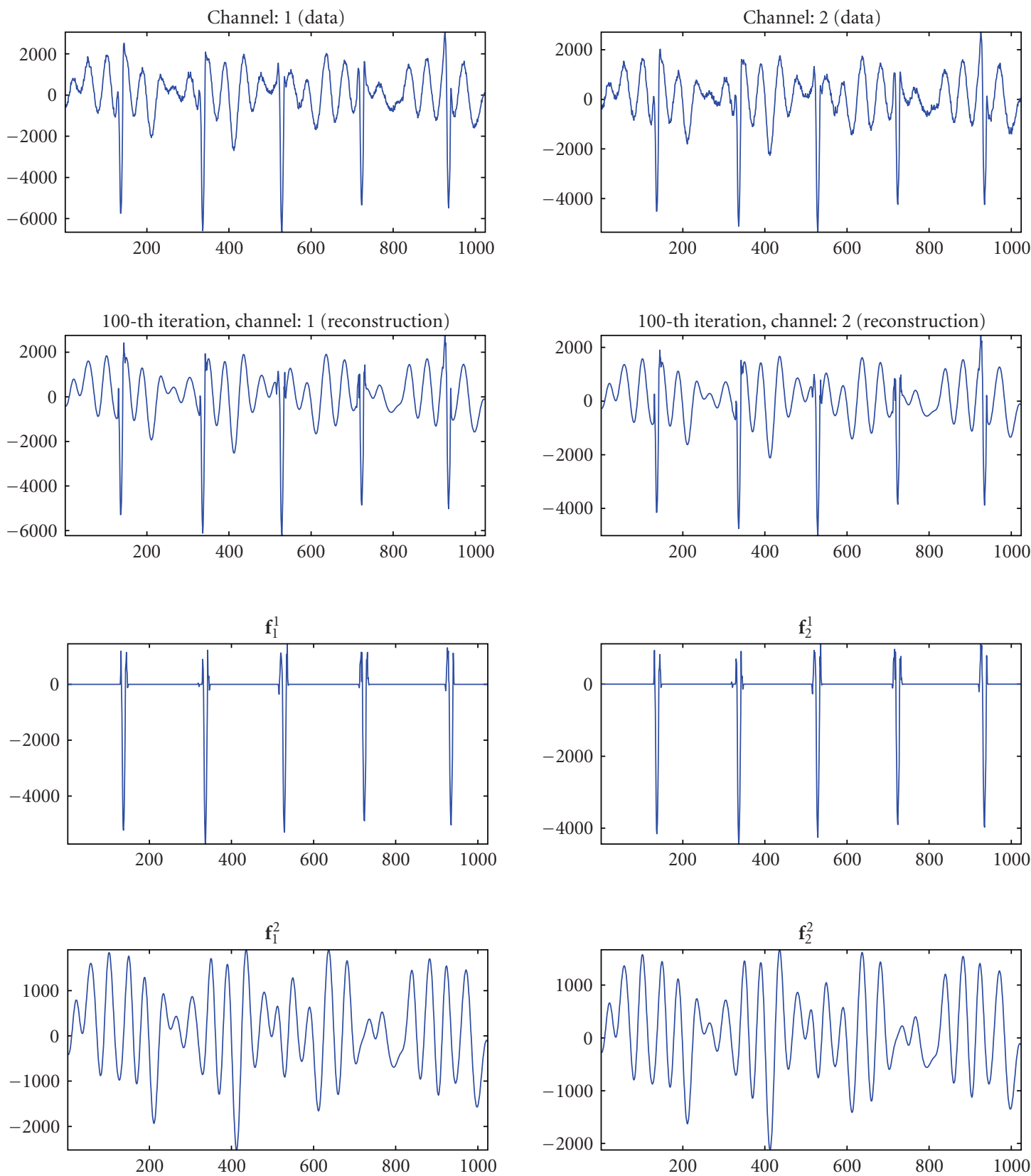

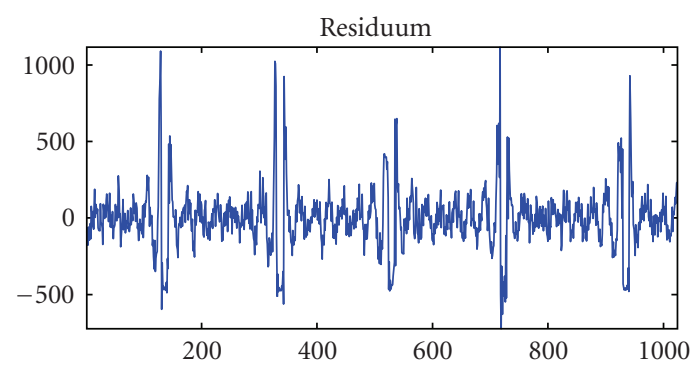

(a)

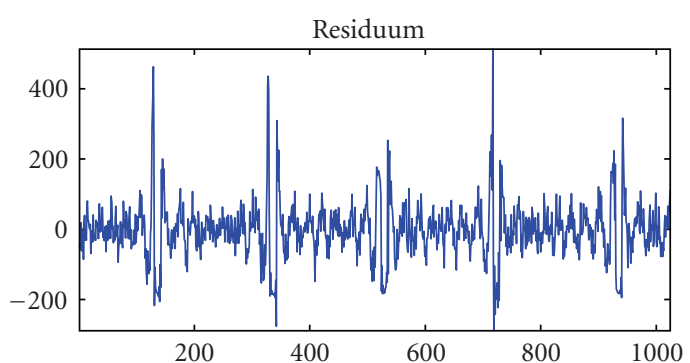

(b)

FIGURE 14: The reconstruction/decomposition of channels 40 (left) and 41 (right). Top row: data to be analyzed (spontaneous activity + sinusoidal signal with maximum $2000 \mathrm{fT}$ ). Second row: reconstructions $\mathbf{f}_{1}^{1}+\mathbf{f}_{1}^{2}$ (left) and $\mathbf{f}_{2}^{1}+\mathbf{f}_{2}^{2}$ (right). Third row: $\mathrm{fMCG}+\mathrm{mMCG}$ reconstructed component $\mathbf{f}_{1}^{1}$ (left) and $\mathbf{f}_{2}^{1}$ (right). Fourth row: MMG + "motion artifacts" reconstructed component $\mathbf{f}_{1}^{2}$ (left) and $\mathbf{f}_{2}^{2}$ (right). Bottom row: residuum (containing noise, contribution of maternal (minor) and fetal heart beat components, and background signals). 
In particular, for the case of $125 \mathrm{fT}$ maximum amplitude, a better reconstruction was achieved (JADE has recovered no sinusoidal signal component at all).

\section{ACKNOWLEDGMENTS}

The authors thank H. Preissl (MEG Center Tübingen, Tübingen, Germany) for encouraging discussions on the signal processing problem and for providing the SQUID data. G. Teschke gratefully acknowledges the partial support by Deutsche Forschungsgemeinschaft Grants TE 354/12, TE 354/3-1, TE 354/4-1, and TE 354/5-1. S. Dahlke gratefully acknowledges the partial support by Deutsche Forschungsgemeinschaft, Grant Da 360/4-3.

\section{REFERENCES}

[1] J. Hykin, R. Moore, K. Duncan, et al., "Fetal brain activity demonstrated by functional magnetic resonance imaging," The Lancet, vol. 354, no. 9179, pp. 645-646, 1999.

[2] R. J. Moore, S. Vadeyar, J. Fulford, et al., "Antenatal determination of fetal brain activity in response to an acoustic stimulus using functional magnetic resonance imaging," Human Brain Mapping, vol. 12, no. 2, pp. 94-99, 2001.

[3] H. Eswaran, H. Preissl, J. D. Wilson, et al., "Short-term serial magnetoencephalography recordings of fetal auditory evoked responses," Neuroscience Letters, vol. 331, no. 2, pp. 128-132, 2002.

[4] H. Eswaran, J. D. Wilson, H. Preissl, et al., "Magnetoencephalographic recordings of visual evoked brain activity in the human fetus," The Lancet, vol. 360, no. 9335, pp. 779-780, 2002.

[5] J. M. Lengle, M. Chen, and R. T. Wakai, "Improved neuromagnetic detection of fetal and neonatal auditory evoked responses," Clinical Neurophysiology, vol. 112, no. 5, pp. 785792, 2001.

[6] U. Schneider, E. Schleussner, J. Haueisen, H. Nowak, and H.-J. Seewald, "Signal analysis of auditory evoked cortical fields in fetal magnetoencephalography," Brain Topography, vol. 14, no. 1, pp. 69-80, 2001.

[7] S. E. Robinson, J. Vrba, and J. McCubbin, "Separating fetal meg signals from the noise," in Biomag 2002, H. Nowak, J. Haueisein, F. Giessler, and R. Huonker, Eds., pp. 665-667, VDE Verlag, Berlin, Germany, 2002.

[8] R. T. Wakai and W. J. Lutter, "Matched-filter template generation via spatial filtering: application to fetal biomagnetic recordings," IEEE Transactions on Biomedical Engineering, vol. 49, no. 10, pp. 1214-1217, 2002.

[9] J. Vrba, S. E. Robinson, J. McCubbin, et al., "Fetal MEG redistribution by projection operators," IEEE Transactions on Biomedical Engineering, vol. 51, no. 7, pp. 1207-1218, 2004.

[10] M. Samonas, M. Petrou, and A. A. Loannides, "Identification and elimination of cardiac contribution in single-trial magnetoencephalographic signals," IEEE Transactions on Biomedical Engineering, vol. 44, no. 5, pp. 386-393, 1997.

[11] P. Strobach, K. Abraham-Fuchs, and W. Harer, "Eventsynchronous cancellation of the heart interference in biomedical signals," IEEE Transactions on Biomedical Engineering, vol. 41, no. 4, pp. 343-350, 1994.

[12] E. J. Candès, J. Romberg, and T. Tao, "Robust uncertainty principles: exact signal reconstruction from highly incomplete frequency information," IEEE Transactions on Information Theory, vol. 52, no. 2, pp. 489-509, 2006.
[13] E. J. Candes and T. Tao, "Near-optimal signal recovery from random projections: universal encoding strategies?" IEEE Transactions on Information Theory, vol. 52, no. 12, pp. 54065425, 2006.

[14] D. L. Donoho, "Compressed sensing," IEEE Transactions on Information Theory, vol. 52, no. 4, pp. 1289-1306, 2006.

[15] E. J. Candès and D. L. Donoho, "New tight frames of curvelets and optimal representations of objects with piecewise $C^{2}$ singularities," Communications on Pure and Applied Mathematics, vol. 57, no. 2, pp. 219-266, 2004.

[16] M. Elad, J.-L. Starck, P. Querre, and D. L. Donoho, "Simultaneous cartoon and texture image inpainting using morphological component analysis (MCA)," Applied and Computational Harmonic Analysis, vol. 19, no. 3, pp. 340-358, 2005.

[17] S. Dahlke, M. Fornasier, H. Rauhut, G. Steidl, and G. Teschke, "Generalized coorbit theory, Banach frames, and the relation to $\alpha$-modulation spaces," Proceedings of the London Mathematical Society, vol. 96, no. 2, pp. 464-506, 2008.

[18] H. G. Feichtinger and K. Gröchenig, "A unified approach to atomic decompositions via integrable group representations," in Proceedings of the US-Swedish Seminar on Function Spaces and Applications, vol. 1302 of Lecture Notes in Mathematics, pp. 52-73, Lund, Sweden, June 1988.

[19] H. G. Feichtinger and K. H. Gröchenig, "Banach spaces related to integrable group representations and their atomic decompositions-I," Journal of Functional Analysis, vol. 86, no. 2, pp. 307-340, 1989.

[20] H. G. Feichtinger and K. H. Gröchenig, "Banach spaces related to integrable group representations and their atomic decompositions_-part II," Monatshefte für Mathematik, vol. 108, no. 2-3, pp. 129-148, 1989.

[21] J. A. Tropp, "Algorithms for simultaneous sparse approximation-part II: convex relaxation," Signal Processing, vol. 86, no. 3, pp. 589-602, 2006.

[22] J. A. Tropp, A. C. Gilbert, and M. J. Strauss, "Algorithms for simultaneous sparse approximation-part I: greedy pursuit," Signal Processing, vol. 86, no. 3, pp. 572-588, 2006.

[23] M. Fornasier and H. Rauhut, "Recovery algorithms for vector valued data with joint sparsity constraints," SIAM Journal on Numerical Analysis, vol. 46, no. 2, pp. 577-613, 2007.

[24] G. Teschke and R. Ramlau, "An iterative algorithm for nonlinear inverse problems with joint sparsity constraints in vector-valued regimes and an application to color image inpainting," Inverse Problems, vol. 23, no. 5, pp. 1851-1870, 2007.

[25] I. Daubechies, G. Teschke, and L. Vese, "Iteratively solving linear inverse problems with general convex constraints," Inverse Problems and Imaging, vol. 1, no. 1, pp. 29-46, 2007.

[26] I. Daubechies, G. Teschke, and L. Vese, "On some iterative concepts for image restoration," Advances in Imaging and Electron Physics, vol. 150, pp. 1-51, 2008.

[27] R. Ramlau and G. Teschke, "A Tikhonov-based projection iteration for nonlinear Ill-posed problems with sparsity constraints," Numerische Mathematik, vol. 104, no. 2, pp. 177203, 2006.

[28] G. Teschke, "Multi-frame representations in linear inverse problems with mixed multi-constraints," Applied and Computational Harmonic Analysis, vol. 22, no. 1, pp. 43-60, 2007. 